\title{
PAPEL DOS FLUXOS DE CALOR LATENTE E SENSÍVEL EM SUPERFÍCIE ASSOCIADO A UM CASO DE CICLOGÊNESE NA COSTA LESTE DA AMÉRICA DO SUL
}

\author{
EVERSON DAL PIVA ${ }^{1}$, MARLEY CAVALCANTE DE LIMA MOSCATI ${ }^{2}$ e \\ MANOEL ALONSO GAN ${ }^{2}$
}

\author{
${ }^{1}$ Centro Regional Sul, Instituto Nacional de Pesquisas Espaciais -CRS/INPE Santa Maria, RS \\ ${ }^{2}$ Instituto Nacional de Pesquisas Espaciais - INPE \\ everson.bento@gmail.com \\ marley@cptec.inpe.br \\ alonso@cptec.inpe.br
}

Recebido Fevereiro 2008 - Aceito Setembro 2008

\section{RESUMO}

Neste estudo foram realizados experimentos numéricos com um modelo meteorológico regional a fim de verificar o impacto dos Fluxos de Calor Latente (FCL) e Calor Sensível (FCS) em superfície no desenvolvimento de um ciclone intenso ocorrido sobre a costa leste da região sudeste da América do Sul, entre os dias 24 e 26 de julho de 1998. A taxa de intensificação do ciclone foi reduzida em 6 $\mathrm{hPa} / 24 \mathrm{~h}$ no experimento em que os FCL e FCS estavam ausentes. Verificou-se que a ausência do FCL apresentou maior impacto do que a ausência do FCS, fazendo com que a taxa de intensificação fosse reduzida em $6 \mathrm{hPa} / 24 \mathrm{~h}$ para o experimento sem FCL e permanecesse inalterada sem FCS, mas neste último a isóbara de menor valor no centro do ciclone ocupou uma área menor. A ausência dos FCL e FCS em superfície gerou uma camada mais seca e mais fria próxima à superfície oceânica, reduzindo a instabilidade potencial do ambiente e diminuindo a intensificação do ciclone. Concluiu-se, portanto que os FCL e FCS em superfície foram importantes antes da fase de mais rápido desenvolvimento do ciclone, agindo no sentido de preparar o ambiente para uma ciclogênese mais intensa, através do fornecimento de energia e umidade para a baixa troposfera. Na fase de mais rápido desenvolvimento, a energia e a umidade disponíveis nas camadas mais baixas da atmosfera (inseridos na fase anterior) também foram importantes, permitindo ao ciclone se desenvolver mais intensamente do que ocorreria em um ambiente mais seco e menos instável termicamente.

Palavras-Chave: ciclogênese, fluxos de calor em superfície, modelo regional.

\begin{abstract}
ROLE OF SURFACE LATENT AND SENSIBLE HEAT FLUXES ASSOCIATED TO A SOUTH AMERICA EAST COAST CYCLOGENESIS CASE

Numeric experiments were run with meteorological regional model with the purpose to verify the impact of both latent and sensible surface heat fluxes (FCL and FCS, respectively) during the development of an intense east cost cyclone over southeastern region of South America from 24 to 26 July, 1998. The intensification rate of the cyclone was reduced by $6 \mathrm{hPa} / 24 \mathrm{~h}$ in the experiment where the FCL and FCS were absents. It was also verified that the absence of FCL presented more impact than without FCS. The intensification rate was reduced by $6 \mathrm{hPa} / 24 \mathrm{~h}$ in the experiment without FCL and became unaltered in the experiment without FCS, but in the later the isobar with minimum value around the cyclone center occupied a smaller area. The absence of the FCL and FCS generated a drier and colder layer near the oceanic surface, reducing the environmental potential instability and decreasing the cyclone intensification. With these results it is possible to conclude that the FCL and FCS were important before the phase of most rapid cyclone development, preparing the environment to a more intense cyclogenesis, supplying both energy and moisture to the lower troposphere. In the phase of the most rapid development, the available energy and moisture (inserted in the previous phase) in the lower levels were also important, allowing the cyclone to develop more intensely than it could be in a drier and less thermically unstable environment.
\end{abstract}

Keywords: cyclogenesis, surface heat flux, regional model. 


\section{INTRODUÇÃO}

Muitas das situações de tempo severo registradas nas regiões Sul e Sudeste do Brasil estão associadas à passagem de ciclones extratropicais intensos, que durante sua trajetória causam muitos prejuízos em função dos ventos fortes e da intensa precipitação que os acompanham. O processo de rápida intensificação de ciclones extratropicais tem sido denominado na literatura de ciclogênese intensa, ciclogênese explosiva ou ciclogênese de costa leste (Miller, 1946; Chen et al., 1992). A expressão "explosiva" diz respeito ao curto intervalo de tempo em que o sistema apresenta grande desenvolvimento, expresso pela taxa de aprofundamento da pressão no centro do ciclone em superfície. Sanders e Gyakum (1980) classificaram como explosiva a ciclogênese que apresenta taxa de intensificação maior ou igual a 1 Bergeron, ou seja, decréscimo da pressão central do sistema de $24 \mathrm{hPa}$ em $24 \mathrm{~h}$, ajustada para a latitude média onde a ciclogênese se desenvolveu.

A ciclogênese de costa leste é quase exclusivamente um fenômeno oceânico (Sanders e Gyakum, 1980; Ogura e Juang, 1990; Juang e Ogura, 1990; Nielsen e Dole, 1992) e ocorre preferencialmente nas porções oeste dos Oceanos Atlântico e Pacífico, onde as características fisiográficas se combinam para produzir condições favoráveis para sua ocorrência. Como mecanismos forçantes para o desenvolvimento de ciclogênese de costa leste, têm-se os efeitos da Liberação de Calor Latente (LCL), dos Fluxos de Calor Total - FCT (definido com sendo a soma dos fluxos em superfície de Calor Sensível (FCS) e Calor Latente (FCL)), da topografia e dos distúrbios na troposfera superior (Sanders e Gyakum, 1980; Nuss e Anthes, 1987; Davis e Emanuel, 1988; Kuo e Reed, 1988; Kuo e Low-Nam, 1990; Wash et al., 1992; Uccellini, 1992), havendo forte interação entre estes processos (Roebber, 1989; Kuo et al., 1991a).

Também se ressalta que a ciclogênese, geralmente é mais intensa em regiões de fortes gradientes de Temperatura da Superfície do Mar (TSM) do que em alto mar (Sanders e Gyakum, 1980; Holland et al., 1987; Sinclair, 1995; Yinlong e Mingyu, 1999) e mais frequentemente ocorre durante a estação de inverno (Sanders e Gyakum, 1980; Gan e Rao, 1991; Sinclair 1995). Este cenário tipicamente inclui intensos fluxos de calor e umidade da superfície do oceano para a atmosfera (Holt e Chang, 1993).

Dentre os mecanismos forçantes citados acima, o papel dos fluxos de calor e de umidade em superfície ainda não são muito bem conhecidos, pois, embora o FCL e FCS sejam importantes na geração de um ambiente pré-ciclogenético favorável ao desenvolvimento explosivo (Bosart e Lin, 1984; Uccelinni et al., 1987; Atlas, 1987; Kuo et al., 1991b), há casos em que estes não foram essenciais para o rápido aprofundamento do sistema (Kuo e Reed, 1988; Kuo e Low-Nam, 1990; Reed e Simmons, 1991; Chang et al., 1996).
Poucos estudos sobre ciclogênese na costa leste da América do Sul têm sido realizados (Necco, 1982; Sinclair, 1995, 1996; Gan e Rao, 1991 e 1996; Bonatti, 2000a,b; Reboita et al., 2005; Mendes et al., 2007), principalmente utilizando modelos numéricos (Bonatti e Rao, 1987; Innocentini e Caetano Neto, 1996; Saraiva, 1996; Piva, 2001; Oda, 2005; Reboita et al., 2007). Além disso, há uma necessidade cada vez maior em melhorar os modelos operacionais quanto à ocorrência e à intensidade dos ciclones desenvolvidos na costa leste, visando principalmente reduzir o impacto sócio-econômico que estes sistemas podem causar em sua trajetória.

Pelo exposto, é de suma importância estudar os processos dinâmicos envolvidos em eventos de ciclogênese com rápido desenvolvimento. Através de estudos numéricos é possível isolar os processos físicos associados, e assim, determinar dentre estes os mais importantes, e em que fase do desenvolvimento estes afetam o sistema em estudo. Com base no exposto, o objetivo deste trabalho é investigar numericamente o papel dos FCS, FCL e FCT no desenvolvimento de um ciclone ocorrido na costa leste da América do Sul, no período de 24 a 26 de julho de 1998, visando determinar em qual estágio do ciclo de vida do ciclone estes fluxos são mais importantes. Este caso de ciclogênese foi escolhido, porque teve um rápido e intenso desenvolvimento (embora não tenha atingido a intensidade de um Bergeron), e também porque a maior parte de sua intensificação foi durante o período em que o ciclone se deslocou do continente para o oceano. Assim, pode-se avaliar o papel dos fluxos de calor em superfície durante a fase de rápida intensificação do ciclone na costa leste da América do Sul.

\section{MATERIAL E MÉTODOS}

\subsection{DADOS}

Os dados utilizados fazem parte do conjunto das reanálises do Projeto R-1 (Kalnay et al., 1996) do National Centers for Environment Prediction / National Center for Atmospheric Research (NCEP/NCAR). Esses dados estão dispostos numa grade global de $2,5^{\circ}$ x $2,5^{\circ}$, em 17 níveis de pressão na vertical. As variáveis consideradas foram as componentes zonal (u) e meridional (v) do vento (expressas em $\left.\mathrm{m} \mathrm{s}^{-1}\right)$, a altura geopotencial ( $\left.\mathrm{z}, \mathrm{em} \mathrm{mgp}\right)$, a temperatura do ar ( $T$, em K), a pressão reduzida ao nível médio do mar (PNMM, em $\mathrm{hPa}$ ), a umidade específica (em $\mathrm{g} \mathrm{kg}^{-1}$ ), o FCL e o FCS (ambos em $\mathrm{W} \mathrm{m}^{-2}$ ), nos horários das 0000, 0600, 1200 e 1800 UTC. Também, a partir dos dados de reanálise, parâmetros derivados foram calculados, como por exemplo a temperatura potencial $(\theta)$ e o parâmetro de estabilidade estática (s). As variáveis $\theta$ e s, são dadas por (Bluestein, 1992): 


$$
\begin{gathered}
\theta=T\left(\frac{1000}{p}\right)^{\frac{R}{c_{p}}} \\
\sigma=-\frac{R T}{p}\left(\frac{\partial \ln \theta}{\partial p}\right)
\end{gathered}
$$

onde $\mathrm{R}$ é a constante dos gases, $\mathrm{c}_{\mathrm{p}}$ é o calor específico à pressão constante, e p é a pressão.

Como condição de contorno sobre os oceanos utilizou-se os dados de TSM dispostos em uma grade de $1^{\circ} \times 1^{\circ}$ (Reynolds e Smith, 1994), obtidos do NCEP/NCAR, os quais consistem da média semanal observada no período de 23/07/1998 a 29/07/1998. As imagens do satélite GOES-8 no canal infravermelho foram disponibilizadas pela Divisão de Satélites Ambientais (DSA)/INPE.

\subsection{Modelo Numérico}

Utilizou-se a versão de equações primitivas na forma de fluxo do Modelo de Área Limitada da Japan Meteorological Agency (MAL-JAPA), desenvolvido por Yamagishi (1980) e Tatsumi (1983), e modificado por Nagata e Ogura (1991). Esse modelo foi adaptado para a região da América do Sul, compreendendo a área entre $100^{\circ} \mathrm{W}$ a $2,5^{\circ} \mathrm{W}$ e $2,5^{\circ} \mathrm{S}$ a $60^{\circ} \mathrm{S}$.

O MAL-JAPA apresenta resolução horizontal de aproximadamente $100 \mathrm{~km}$, com grade do Tipo B de Arakawa. $\mathrm{Na}$ vertical foram utilizadas 16 camadas em coordenada sigma, sendo quatro delas abaixo de $900 \mathrm{hPa}$ e o topo em $100 \mathrm{hPa}$. Nos seis pontos mais externos da grade horizontal, foi aplicado um processo de relaxação entre os campos gerados pelo modelo e os campos da reanálise do NCEP/NCAR. Os processos de condensação considerados foram a Precipitação Acumulada de
Grande Escala (PAGE) e a Precipitação Acumulada Convectiva (PAC). Esta última é parametrizada usando o Esquema Kuo (Kuo, 1974), modificado por Geleyn (1985). O modelo calcula primeiramente a PAC, que depende da instabilidade atmosférica e da convergência de umidade na camada, sendo a precipitação gerada suficiente para levar a estratificação atmosférica a uma condição neutra. Em seguida, o modelo calcula a PAGE, que depende da supersaturação do ambiente. A soma entre a PAC e a PAGE resulta na Precipitação Acumulada Total (PAT). Os processos na camada limite foram avaliados usando o modelo de fechamento de nível 2 de Mellor e Yamada (1974), enquanto os fluxos turbulentos em superfície foram estimados com base na teoria da similaridade de Monin-Obukov, com as funções universais definidas por Bussinger et al. (1971). Informações adicionais sobre o modelo MAL-JAPA são dadas em Innocentini e Caetano Neto (1996) e em Rocha (2000).

\subsection{METODOLOGIA}

Visando avaliar a importância dos FCS, FCL e FCT durante o desenvolvimento do ciclone, selecionou-se um caso de ciclogênese que apresentou a maior parte da sua intensificação no período em que o ciclone se deslocou do continente para o oceano. A tendência de PNMM no centro do ciclone foi usada como uma medida de intensificação (Sinclair, 1994; 1995; Yoshida e Asuma, 2004), embora nem sempre este parâmetro produza uma indicação clara de intensificação do sistema, pois o ciclone pode deslocar-se rapidamente para o pólo e encontrar pressão atmosférica do ambiente cada vez menor. Assim, a tendência da queda de pressão não é necessariamente devido à sua intensificação, mas apenas devido ao seu deslocamento para uma região onde a PNMM local é menor (Holt e Chang, 1993).

O critério utilizado para identificar se o ciclone possuía rápida intensificação foi o de Sanders e Gyakum (1980), no qual só é considerado um ciclone de rápida intensificação quando o decréscimo de pressão no centro do ciclone for igual ou maior a 1 Bergeron, sendo este valor obtido pela expressão:

Tabela 1 - Experimentos realizados.

\begin{tabular}{|c|c|c|}
\hline Experimento & FCS & FCL \\
\hline CON & Presente & Presente \\
\hline SFCS & Ausente & Presente \\
\hline SFCL & Presente & Ausente \\
\hline SFCT & Ausente & Ausente \\
\hline
\end{tabular}




$$
1 \text { Bergeron }=\frac{24 h P a}{24 h} \times\left[\frac{\operatorname{sen} 36^{\circ}}{\operatorname{sen} 60^{\circ}}\right]
$$

onde: $36^{\circ} \mathrm{S}$ é a latitude média da trajetória do ciclone durante o período de $24 \mathrm{~h}$ de intensificação, e $60^{\circ}$ é a latitude de referência. Portanto, o ciclone para ser considerado explosivo deve apresentar um aprofundamento de $16,3 \mathrm{hPa} / 24 \mathrm{~h}$.

A Figura 1 apresenta as imagens de satélite no canal infravermelho para o período de 24 a 26 de julho de 1998. As $24 \mathrm{~h}$ de mais rápido aprofundamento do ciclone ocorreram
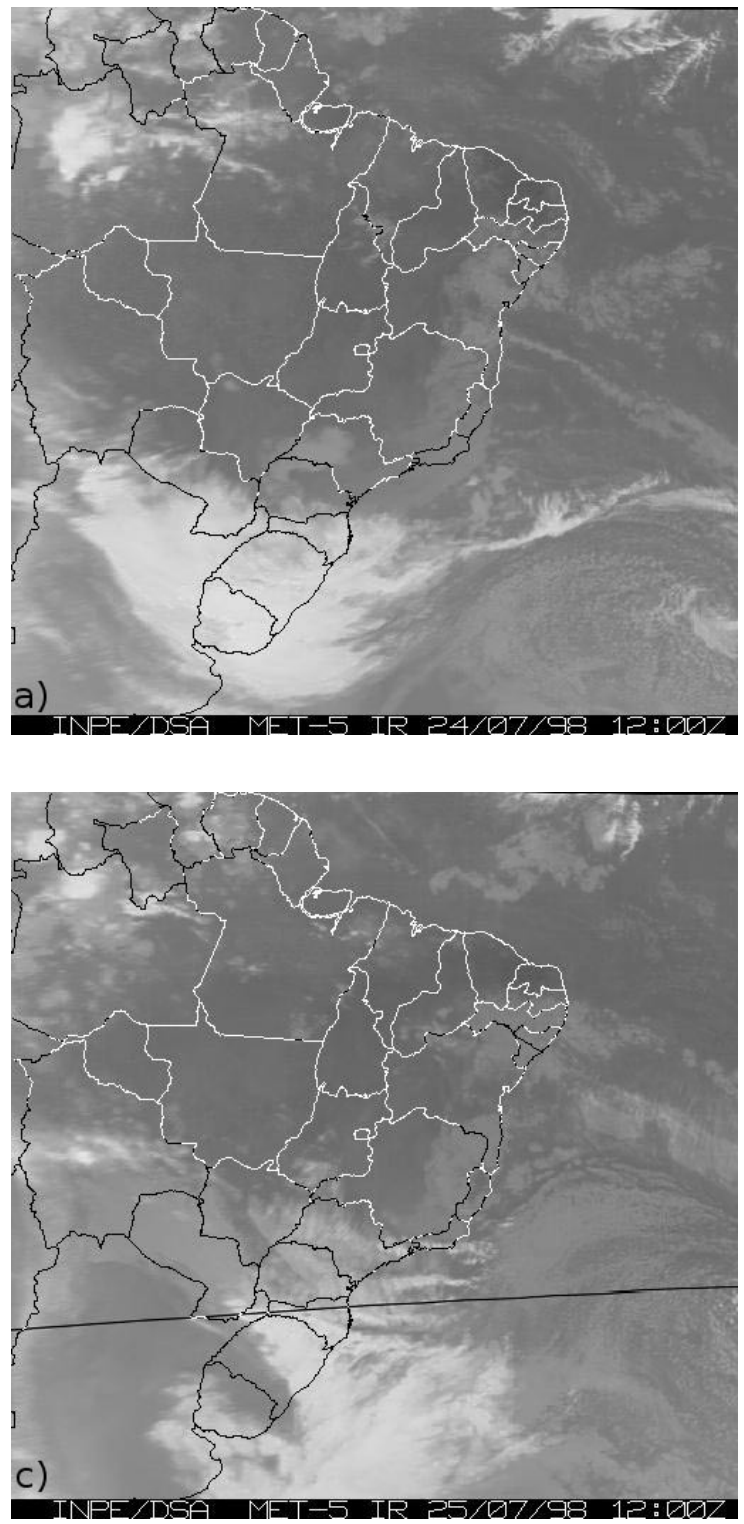

entre as 0000UTC do dia 25/07/1998 e as 0000 UTC do dia 26/07/1998 e foi da ordem de $10 \mathrm{hPa} / 24 \mathrm{~h}$, segundo dados da reanálise do NCEP/NCAR. Segundo o critério de Sanders e Gyakum (1980), este ciclone não pode ser classificado como explosivo, entretanto foi escolhido porque apresentou sua fase de mais rápida intensificação quando passou do continente para o oceano. Deve-se destacar que a reanálise do NCEP/NCAR tem resolução horizontal de $2,5^{\circ}$ de latitude $\times 2,5^{\circ}$ de longitude e, portanto pode subestimar o valor da pressão no centro do ciclone.

Em seguida, realizaram-se simulações com início da rodada $24 \mathrm{~h}$ antes do período de mais rápido desenvolvimento
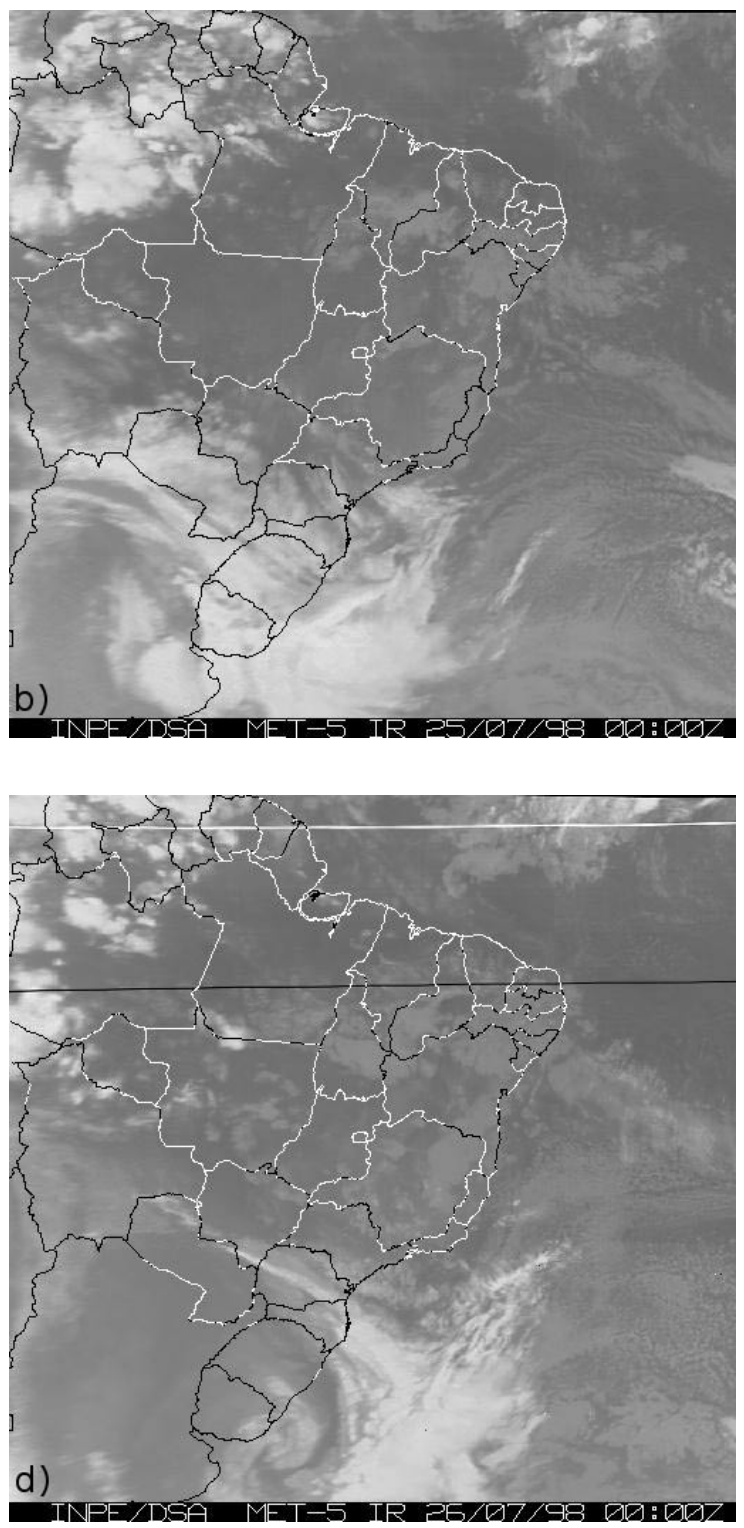

Figura 1 - Imagens do satélite GOES-8 no canal infravermelho, para: a) 24/07/1998 as 1200 UTC, b) 25/07/1998 as 0000 UTC, c) 25/07/1998 as 1200 UTC, d) 26/07/1998 as 0000 UTC. 

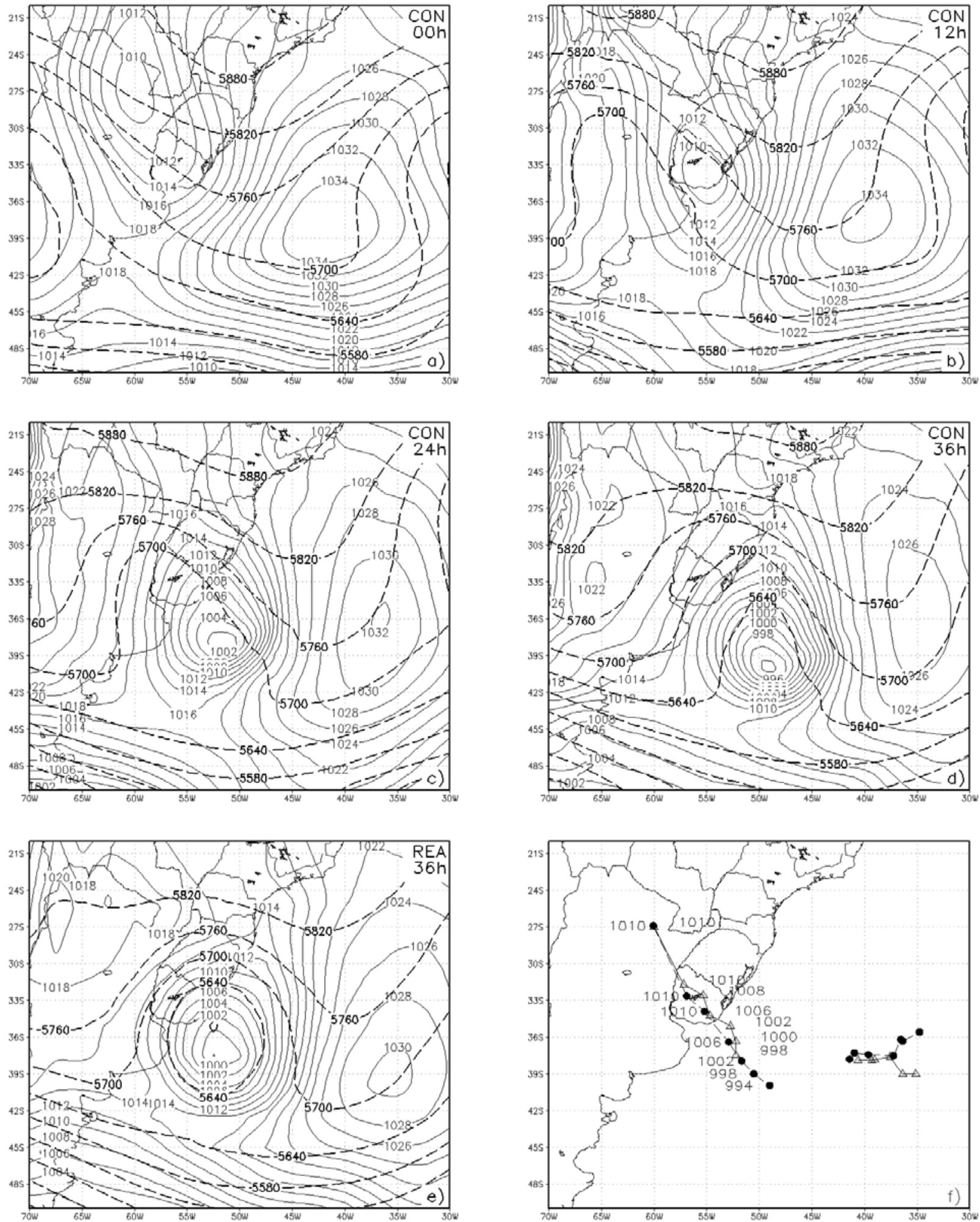

Figura 2 - PNMM (hPa, em intervalos de $2 \mathrm{hPa}$, linha sólida) e altura geopotencial (mgp, em intervalos de 60 mgp, linha tracejada) em $500 \mathrm{hPa}$ no EXP CON para: a) 00 h, b) 12 h, c) 24 h, d) 36 h (d) de simulação, e da reanálise (e) no tempo correspondente a 36 h de simulação; f) Trajetórias e PNMM no centro do ciclone (iniciando-se sobre o continente) e do anticiclone (sobre o oceano) como observado (linha contínua com triângulos) e como simulado pelo EXP CON (linha tracejada com círculos preenchidos). 
do sistema. Foram realizados três experimentos (EXP) numéricos, alterando-se os fluxos de calor em superfície, os quais foram comparados com o EXP de CONtrole (CON), como indicados na Tabela 1. Cada experimento numérico foi integrado por $36 \mathrm{~h}$, com início as 1200UTC do dia 24/07/98, pois no EXP CON iniciado às 0000 UTC do dia 24 , o ciclone não se desenvolveu apropriadamente. Os efeitos dos fluxos de calor em superfície foram avaliados através da diferença entre os campos meteorológicos simulados pelo EXP CON e pelos três EXP sem fluxos de calor em superfície, denominados de SFCL, SFCS e SFCT, para representar os EXP sem FCL, sem FCS e sem FCT, respectivamente.
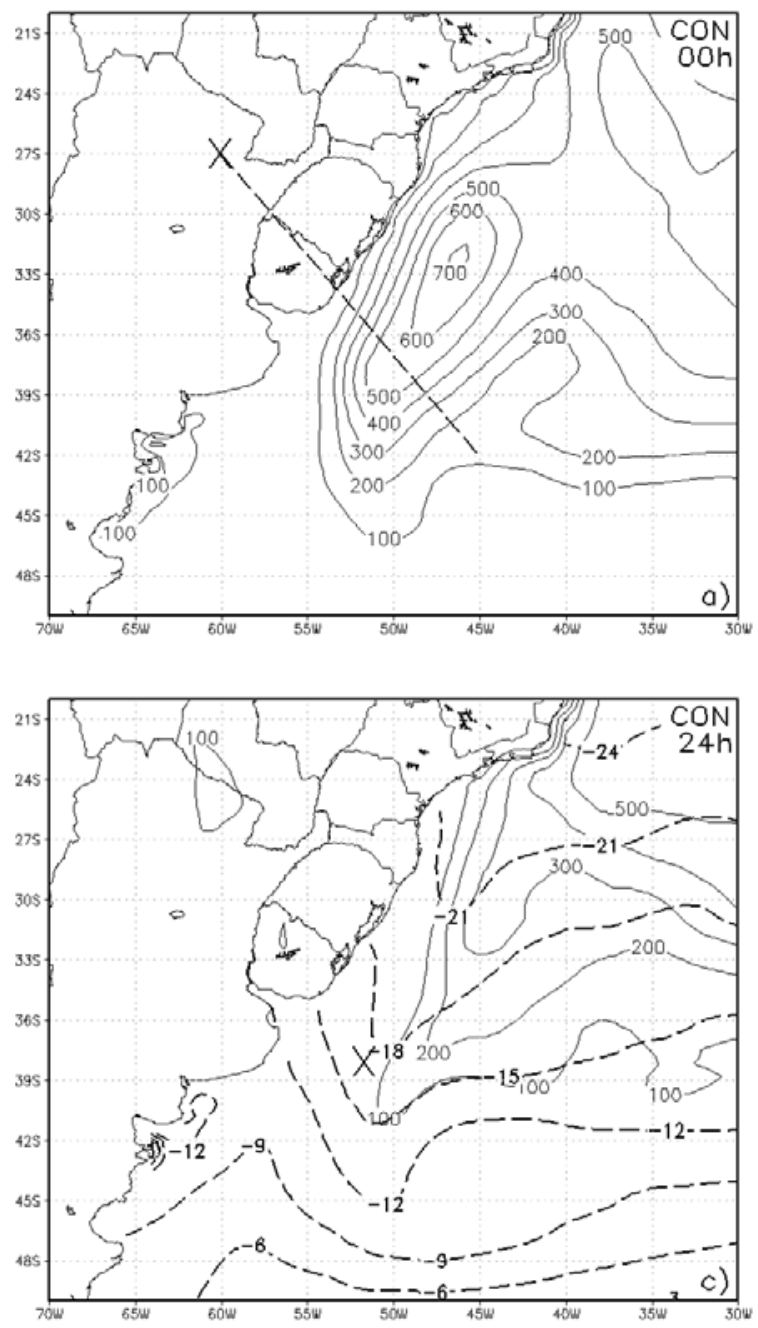

\section{RESULTADOS E DISCUSSÃO}

O EXP CON conseguiu simular o ciclone de forma semelhante ao apresentado pelas reanálises (figuras não apresentadas). Desta forma, a descrição deste caso será feita através dos campos do EXP CON e, quando houver diferenças entre estes resultados e aqueles obtidos com as reanálises, estas serão apresentadas e discutidas.

\subsection{EXPERIMENTO DE CONTROLE}

A simulação do campo de PNMM no EXP CON durante as 36h é mostrada na Figura 2. As 00h da simulação (Figura
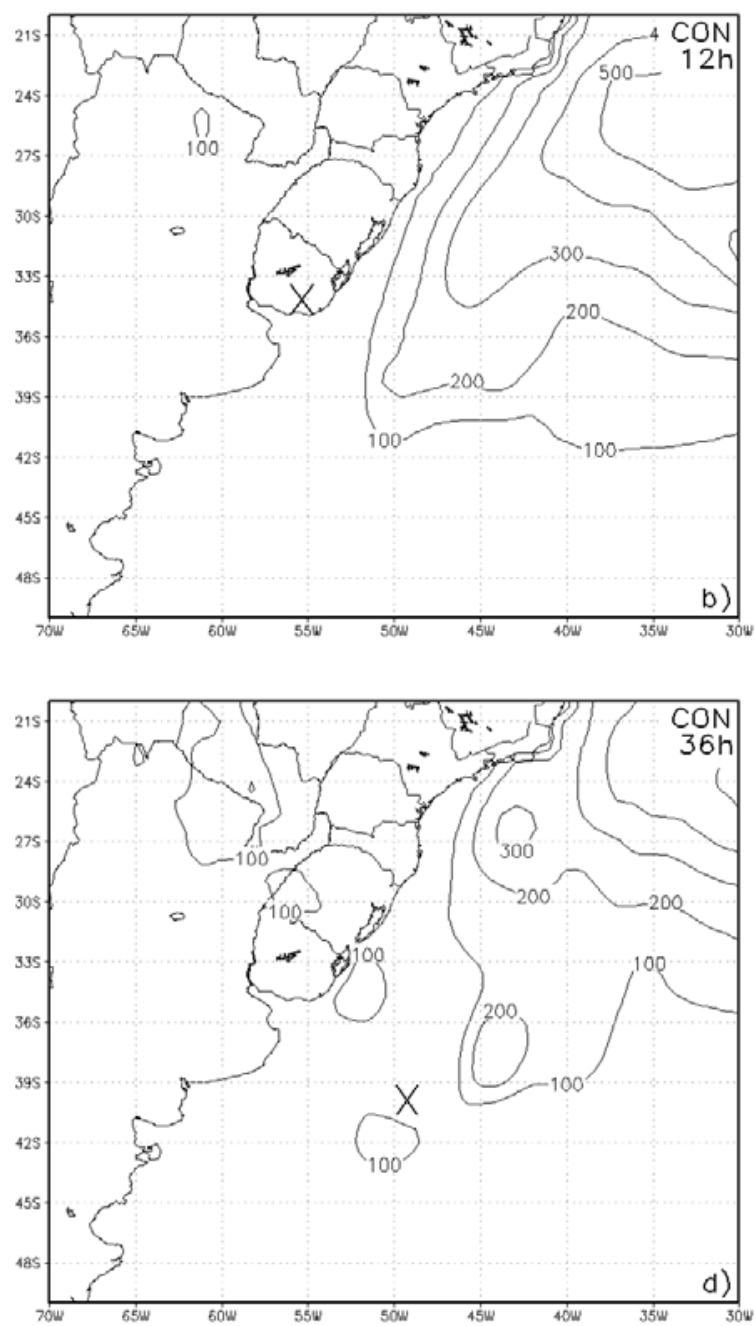

Figura 3 - FCT (em W m ${ }^{-2}$, em intervalos de $100 \mathrm{~W} \mathrm{~m}^{-2}$ ) no EXP CON para as $00 \mathrm{~h}$ (a), $12 \mathrm{~h} \mathrm{(b),} 24 \mathrm{~h}$ (c) e $36 \mathrm{~h}$ (d) de simulação. Tracejado em (c) mostra o campo de TSM $\left({ }^{\circ} \mathrm{C}\right.$, em intervalos de $\left.3^{\circ} \mathrm{C}\right)$ semanal observada. Símbolo X indica o centro do ciclone em superfície. Linha tracejada as $00 \mathrm{~h}$ (a) indica a orientação das seções verticais apresentadas neste artigo. 
2a), verificou-se uma região de baixa PNMM sobre o norte da Argentina, da ordem de $1010 \mathrm{hPa}$, e um centro de alta pressão sobre o Oceano Atlântico Sul, da ordem de $1034 \mathrm{hPa}$. Após $12 \mathrm{~h}$ de simulação (Figura $2 \mathrm{~b}$ ), esta área de baixa pressão se estendeu para sudeste e um ciclone se formou sobre o Uruguai, iniciando-se a fase de mais rápido desenvolvimento do sistema. Nas 24 h seguintes (Figuras 2c-d), as taxas de intensificação do ciclone e de desintensificação do anticiclone foram da ordem de $16 \mathrm{hPa} / 24 \mathrm{~h}$ e $8 \mathrm{hPa} / 24 \mathrm{~h}$, respectivamente. Portanto, na simulação do campo de PNMM, a taxa de intensificação do ciclone foi próxima ao valor da ciclogênese explosiva, como proposta por Sanders e Gyakum (1980), a qual ajustada para a latitude de $36^{\circ} \mathrm{S}$ corresponde a $16,3 \mathrm{hPa} / 24 \mathrm{~h}$.
Nos dados de reanálise, a intensificação do ciclone foi da ordem de $10 \mathrm{hPa} / 24 \mathrm{~h}$ (figura não apresentada) e $\mathrm{o}$ enfraquecimento do anticiclone foi de $4 \mathrm{hPa} / 24 \mathrm{~h}$. A Figura $2 \mathrm{f}$ ilustra a trajetória seguida pelo centro do ciclone (círculos fechados a esquerda) e anticiclone simulada (círculos fechados a direita) e observada na reanálise (triângulos abertos). O valor da PNMM no centro do ciclone está colocado à esquerda da trajetória para o EXP CON e à direita da trajetória para a reanálise. A mais baixa PNMM no centro do ciclone foi de 994 hPa na simulação CON (Figura 2d) e de 998 hPa considerando os dados da reanálise (Figura 2e). Comparando-se os resultados apresentados na Figura 2 com os campos de reanálises (não mostrados), verificou-se as seguintes diferenças nos campos
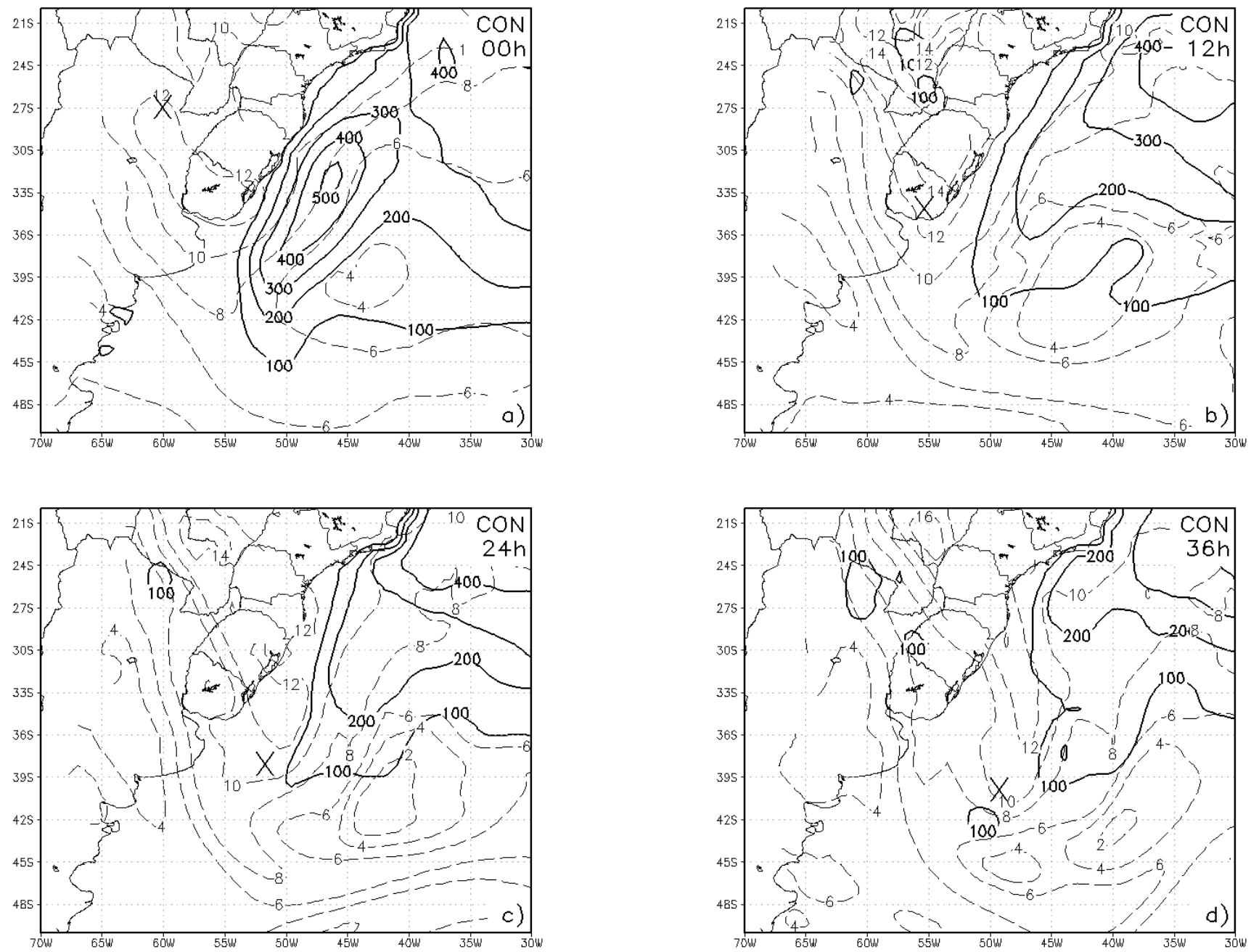

Figura 4-FCL ( $\mathrm{Wm}^{-2}$, em intervalos de $\left.100 \mathrm{Wm}^{-2}\right)$ simulados e observados para as $00 \mathrm{~h}(\mathrm{a}, \mathrm{e}), 12 \mathrm{~h}(\mathrm{~b}, \mathrm{f}), 24 \mathrm{~h}(\mathrm{c}, \mathrm{g})$ e $36 \mathrm{~h}(\mathrm{~d}, \mathrm{~h})$. Tracejado fraco indica umidade específica (em $\mathrm{gkg}^{-1}$, em intervalos de $2 \mathrm{gkg}^{-1}$ ) em $950 \mathrm{hPa}$. Símbolo X indica o centro do ciclone em superfície. 

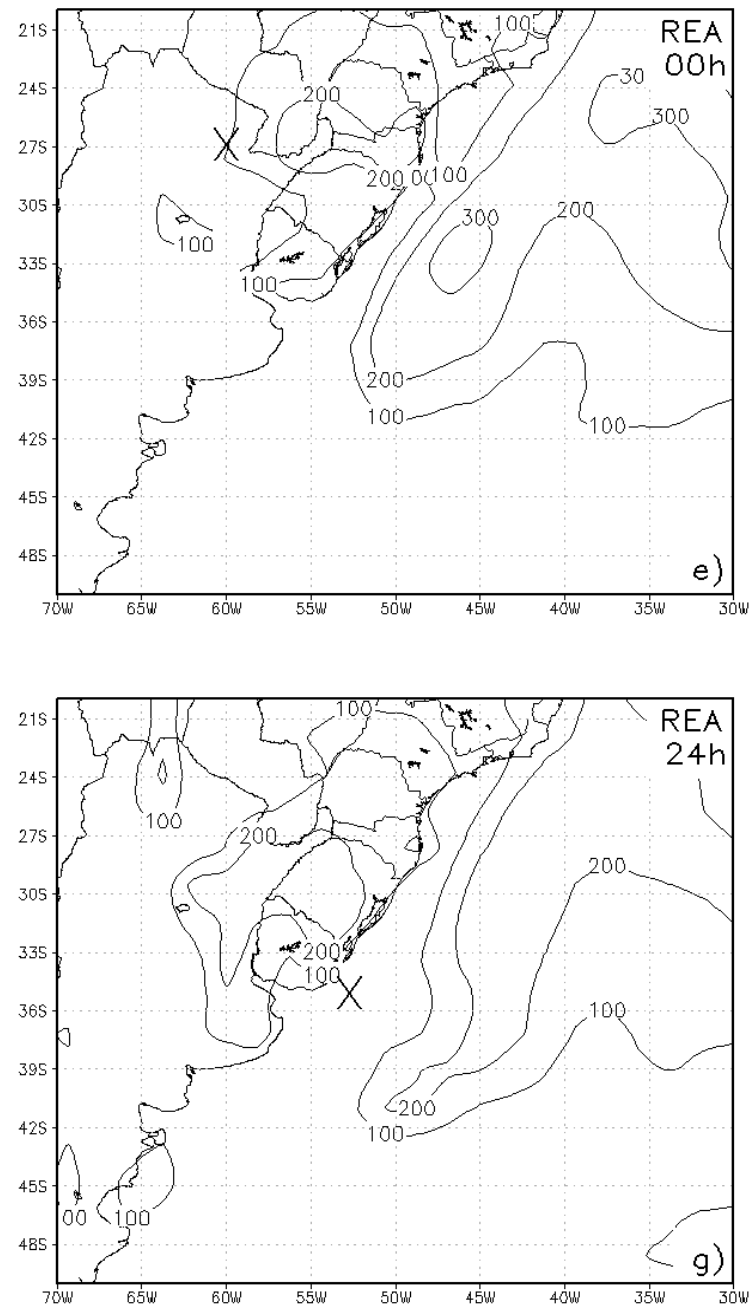

Figura 4 - (Continuação)

de PNMM: a) após 36 h, o EXP CON gerou um ciclone mais intenso e um anticiclone menos intenso do que o observado, ambos com $4 \mathrm{hPa}$ de diferença entre o CON e o observado; b) o EXP CON mostrou a trajetória do ciclone mais regular e para sudeste do que o observado. No caso do anticiclone, tanto no campo de PNMM simulado como no observado, verifica-se que o sistema deslocou-se para norte até $18 \mathrm{~h}$ de simulação e, após esse horário, o anticiclone apresentou trajetória distinta; c) o EXP CON simulou o ciclone com uma inclinação na vertical para oeste maior do que observado na reanálise. Os itens a e b mostram que o Modelo MAL-JAPA tende a intensificar o ciclone em uma taxa maior e a posicioná-lo mais a oeste do que na reanálise. Esta é uma característica do modelo utilizado (Innocentini e Caetano Neto, 1996; Rocha, 2000), assim como de vários outros modelos regionais estudados por Gyakum et al. (1996). Estes estudos não abordaram os motivos para as tendências sistemáticas apresentadas pelos modelos regionais, mas Gyakum et al. (1996) sugerem que pode ser devido à
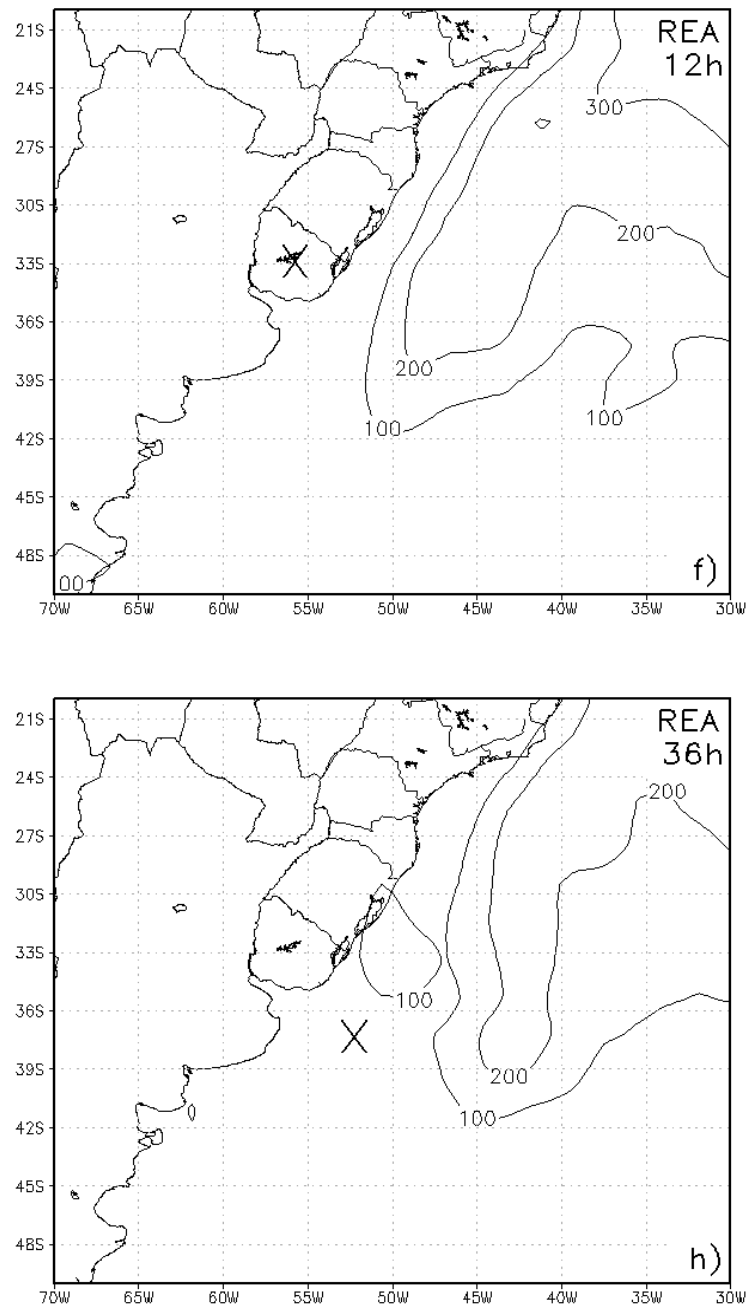

excessiva estabilização da baixa troposfera ao longo da trajetória do ciclone. Baseado na comparação entre esses resultados, concluiu-se que a simulação com o EXP CON reproduziu bem a posição e a intensidade do ciclone durante o seu desenvolvimento.

O FCT simulado pelo MAL-JAPA foi máximo (com centro da ordem de $700 \mathrm{Wm}^{-2}$ ) no início da simulação (as $00 \mathrm{~h}$ ), próximo à costa sul do Brasil (Figura 3a). Os valores positivos indicam fluxo de baixo para cima, ou seja, do solo/oceano para a atmosfera. Na região de deslocamento do ciclone, os FCT se reduziram em magnitude e em área (Figuras $3 b$ e $3 c$ ), tornando-se da ordem de $100-200 \mathrm{Wm}^{-2}$ após 36 $\mathrm{h}$ de simulação, sendo o máximo localizado no lado quente do ciclone (Figura 3d). A relação entre os FCT e o campo de TSM pode ser observada na Figura 3c. A análise dessa figura mostra o avanço para o sul, ao longo da costa brasileira, de águas mais quentes associadas à Corrente do Brasil e, ao longo da costa da Argentina, o avanço para o norte de águas mais frias associadas à Corrente das Malvinas. Devido a este 
padrão de escoamento de águas superficiais, estabelece-se um forte gradiente de $\mathrm{TSM}$ próximo a $43^{\circ} \mathrm{W} ; 38^{\circ} \mathrm{S}$ e uma região de águas quentes próximo à costa do sul do Brasil (Figura 3c).

O padrão de TSM tem forte influência no padrão de FCL e FCS como mostram as Figura 3, 4 e 5. Em geral, aproximadamente $70 \%$ dos FCT corresponderam à contribuição do FCL (Figura 4) e os 30\% restantes corresponderam à contribuição do FCS (Figura 5). Comparando-se a simulação com a reanálise (Figuras 4 e 5), verifica-se que os fluxos de calor gerados pelo modelo foram melhores simulados sobre o oceano do que sobre o continente. Nota-se que as magnitudes do FCL e FCS são superestimadas pelo modelo às 00h (Figuras 4a e 5a, respectivamente), mas no restante da simulação a magnitude de ambos os fluxos é subestimada. Apesar destas discrepâncias, a evolução no tempo dos FCL e FCS foi bem representada, principalmente na região oceânica por onde o ciclone se deslocou.

A evolução dos campos do FCT, assim como dos FCL e FCS, pode ser explicada pela análise dos campos simulados de umidade específica e de $\theta$ em $950 \mathrm{hPa}$ mostrados em tracejado nas Figuras 4 e 5, respectivamente. No início da simulação, os fluxos em superfície foram maiores na costa sul do Brasil, pois, se estabeleceu um escoamento de norte indo do continente para o oceano entre $27^{\circ} \mathrm{S}$ e $36^{\circ} \mathrm{S}$. Este escoamento transportou ar seco (umidade específica de $8 \mathrm{gkg}^{-1}$ ) e não tão quente $(\theta$ de $288 \mathrm{~K}$ ) da região central e sudeste do Brasil para o oceano (Figuras 4a e 5a, respectivamente). Durante o desenvolvimento
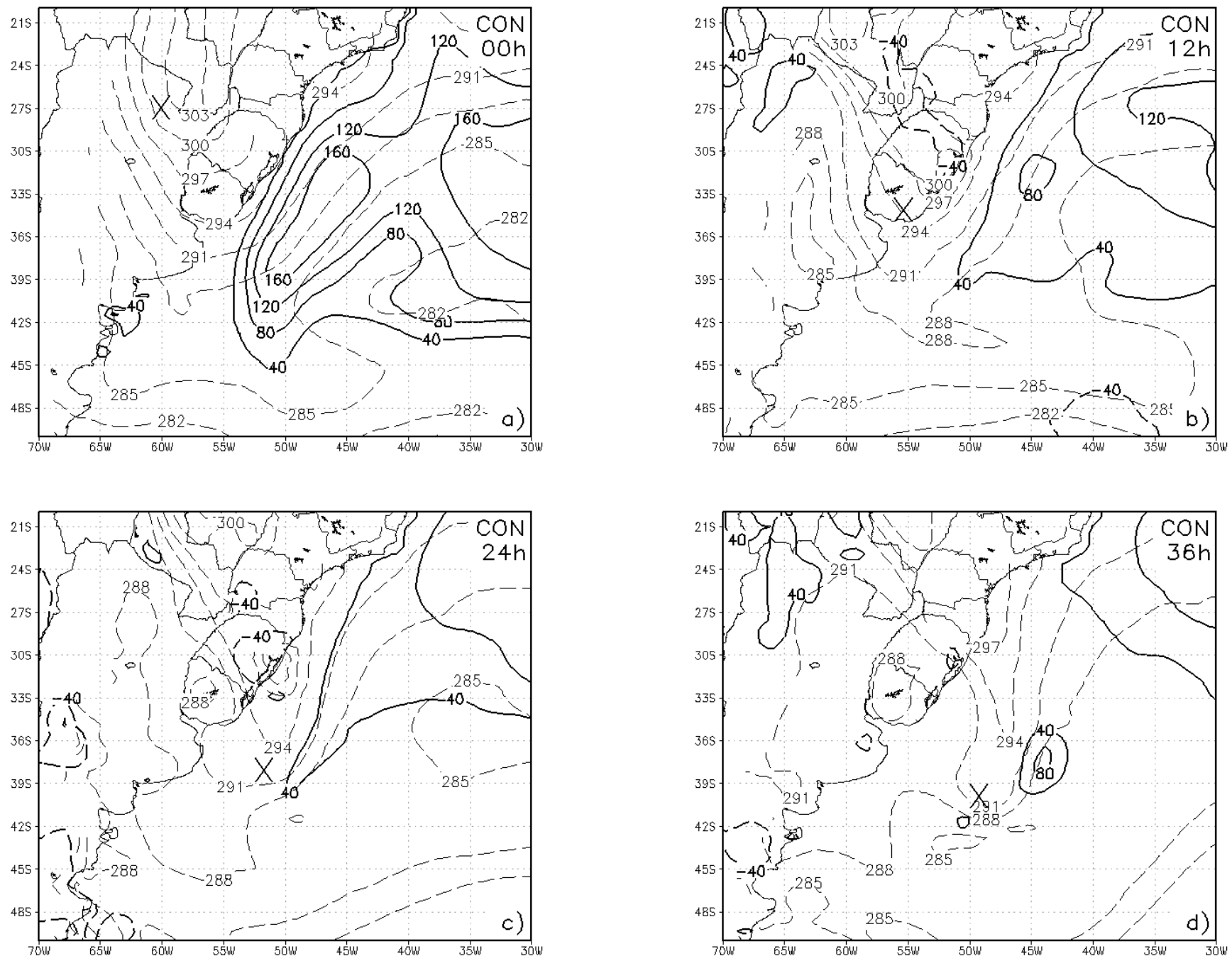

Figura 5 - Como na Figura 4, mas para o FCS (em intervalos de $40 \mathrm{Wm}^{-2}$ ). Tracejado fraco representa a temperatura potencial (K, em intervalo de $3 \mathrm{~K}$ ) em $950 \mathrm{hPa}$. 

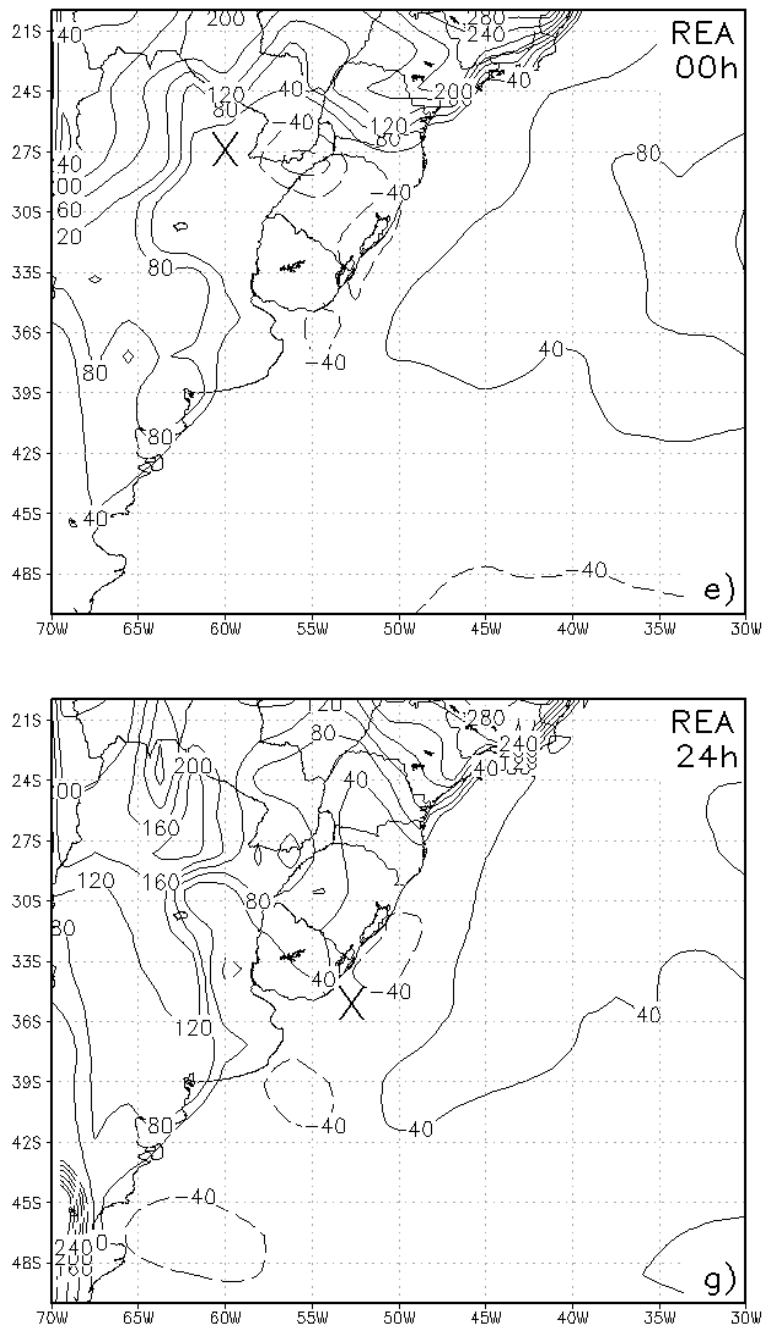

Figura 5 - (Continuação).

do ciclone, o escoamento sobre a costa da região sul do Brasil passou a ser de noroeste, trazendo ar mais quente ( $\theta$ de $294 \mathrm{~K}$ ) e mais úmido (umidade específica de $12 \mathrm{~g} \mathrm{~kg}^{-1}$ ) proveniente da região Amazônica (Figuras 4b-d e 5b-d, respectivamente). Desta forma, os fluxos de calor em superfície se reduziram em magnitude e em área, gerando inclusive valores negativos de FCS (da atmosfera para o oceano/solo) sobre a costa e continente do sul do Brasil (Figuras $5 \mathrm{~b}$ e $5 \mathrm{c}$ ). O escoamento de noroeste de ar quente e úmido é determinante na intensificação dos ciclones extratropicais, que se desenvolvem sobre a América do Sul, como mostrado por Mendes et al. (2007).

\subsection{EXPERIMENTOS NUMÉRICOS: EFEITO DOS FLUXOS DE CALOR EM SUPERFÍCIE}

Os efeitos da ausência dos FCT podem ser visualizados na Figura 6, que mostra o campo de diferença de PNMM
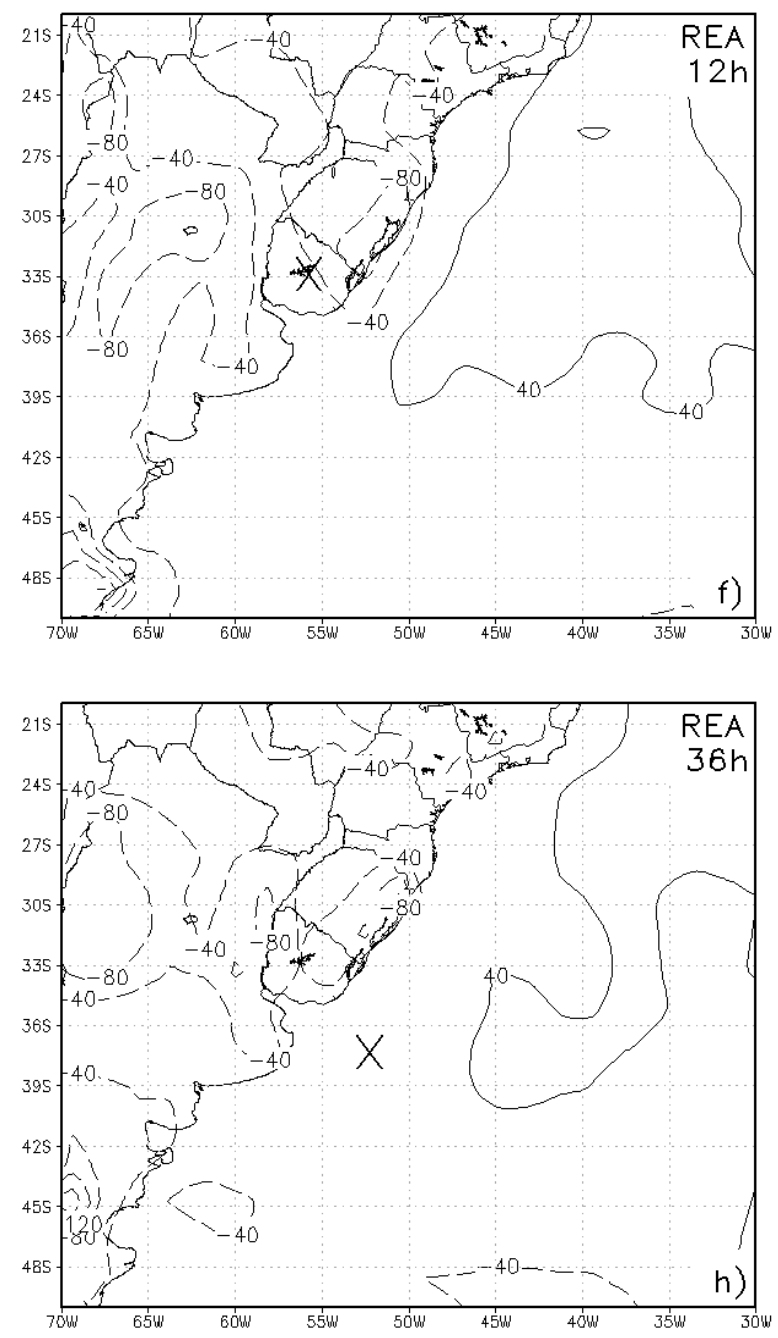

entre o EXP CON e o EXP SFCT, para $24 \mathrm{~h}, 30 \mathrm{~h}$ e $36 \mathrm{~h}$ de simulação. Os valores são negativos e aumentam (em módulo) com o tempo de simulação, indicando que os FCT contribuem para diminuir a pressão em superfície. Os maiores efeitos do FCL e do FCS foram observados nos setores central, sul e leste do ciclone, sendo o máximo de $-5 \mathrm{hPa}$ após $36 \mathrm{~h}$ de simulação (Figura 6c). A ausência dos FCT gerou um ciclone com PNMM central de $1000 \mathrm{hPa}$ (Figura $6 \mathrm{~d}$ ), $6 \mathrm{hPa}$ superior a do EXP CON (Figura 2d). Assim, pode-se verificar que na ausência dos FCT o ciclone ficou menos intenso e a taxa de desenvolvimento reduziu de $16 \mathrm{hPa} / 24 \mathrm{~h}$ para $10 \mathrm{hPa} / 24$ $\mathrm{h}$, se distanciando da categoria de ciclogênese explosiva.

A ausência dos FCL gerou diferenças de PNMM da ordem de $-7 \mathrm{hPa}$ no fim da simulação (Figura 7a) e a PNMM no centro do ciclone foi de $1000 \mathrm{hPa}$ (Figura 7b). No EXP SFCS (Figura $7 \mathrm{c}, \mathrm{d}$ ), a diferença de PNMM em torno do ciclone foi pequena $(-1 \mathrm{hPa})$ e a pressão no centro do ciclone foi a mesma 
que no EXP CON (Figura 2d), embora a área do contorno de $994 \mathrm{hPa}$ tenha sido menor. Interessante notar que o impacto dos FCL foi superior ao impacto dos FCT, o qual representa a soma do FCL e FCS. A explicação para esta aparente contradição se encontra no fato que os valores de FCS foram negativos sobre o Rio Grande do Sul e seu litoral (Figura 5), ou seja, da atmosfera para a superfície, resfriando a troposfera inferior como será mostrado mais adiante. Isso significa que no EXP SFCL, além da ausência do fluxo de umidade (FCL ausente) e seu impacto na LCL, tem-se também FCS com valores negativos, os quais resfriam a troposfera inferior, aumentando assim a estabilidade atmosférica; explicando porque que no EXP SFCL o ciclone foi menos intenso do que no EXP SFCT. Ressalta-se que, mesmo que a ausência dos
FCT tenha gerado diferenças de PNMM menores do que a ausência dos FCL, a pressão reduzida no centro do ciclone foi de $1000 \mathrm{hPa}$ para ambos os experimentos. Isto ocorre porque a máxima diferença de PNMM não foi registrada no centro do ciclone, e sim à sudeste na área da frente quente. Observa-se que no centro do ciclone, tanto na ausência de FCL quanto de FCT, a diferença de PNMM foi da ordem de $5 \mathrm{hPa}$ (Figuras $6 \mathrm{~b}$ e 7a). Ressalta-se que o horário de início da ciclogênese não foi modificado com a ausência dos fluxos, ou seja, em todos os experimentos o ciclone iniciou as 1800 UTC do dia 24.

Os efeitos da ausência dos FCT no campo de $\theta$ em 950 $\mathrm{hPa}$ pode ser visto na Figura 8, que mostra a diferença entre o EXP CON e EXP SFCT. A ausência dos FCT em superfície gerou diferenças positivas de $\theta$ na maior parte do domínio, as
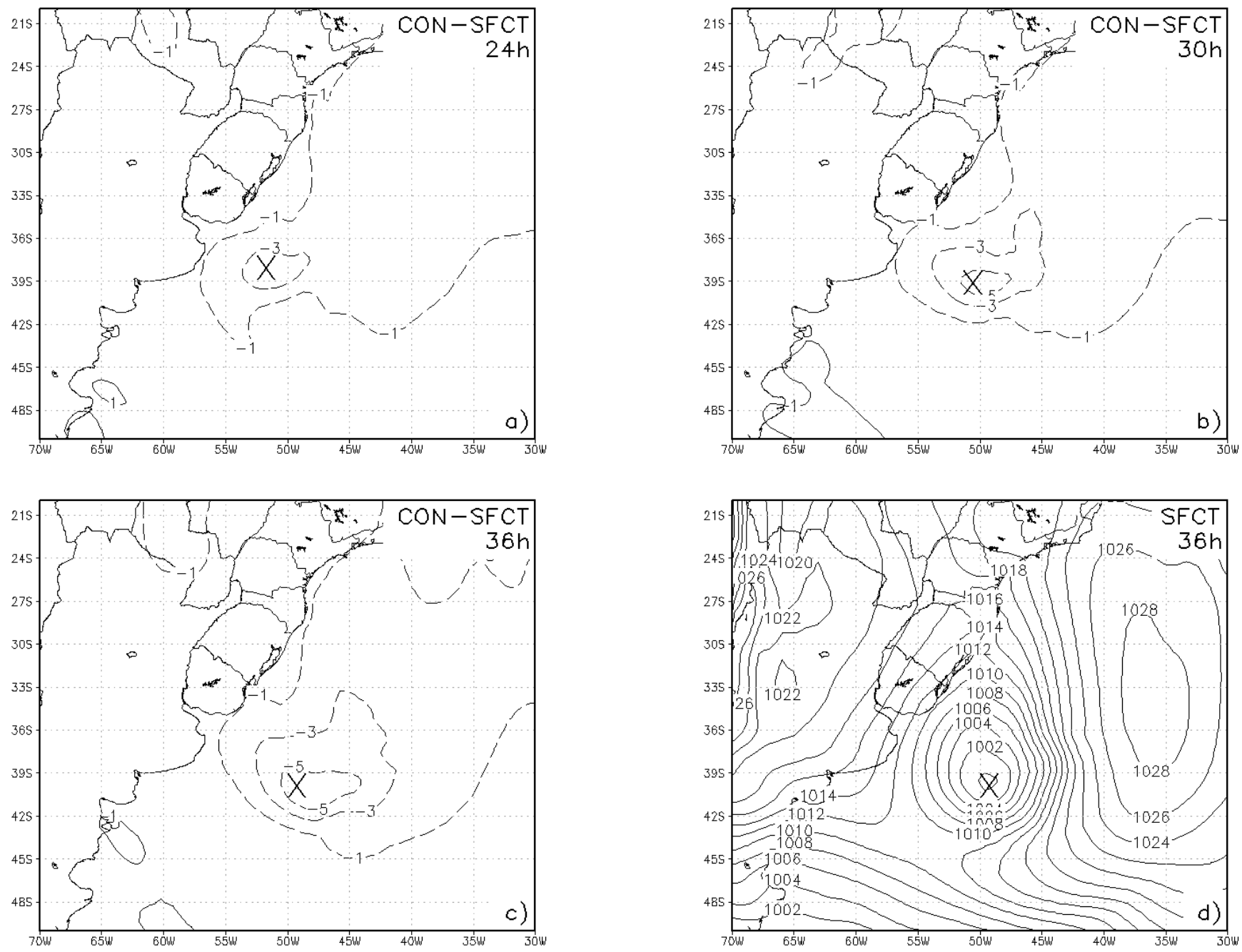

Figura 6 - Diferença de PNMM (hPa, em intervalos de $2 \mathrm{hPa}$ ) entre o EXP CON e EXP SFCT para as $24 \mathrm{~h}(\mathrm{a}), 30 \mathrm{~h}$ (b) e $36 \mathrm{~h}$ (c) de simulação. PNMM ( $\mathrm{hPa}$, em intervalos de $2 \mathrm{hPa}$ ) para as $36 \mathrm{~h}$ de simulação no EXP SFCT (d). O símbolo X indica o centro do ciclone em superfície. 
quais foram maiores na região do ciclone, sendo o máximo de $9 \mathrm{~K}$ a leste do centro do ciclone (Figuras 8a-c). Diferenças negativas de FCT foram observadas sobre o continente e no litoral gaúcho nas primeiras $24 \mathrm{~h}$ de simulação (Figuras 8a-b), indicando que os FCT resfriaram a troposfera inferior nestas regiões. Separando-se as contribuições dos FCL e FCS (Figura 9), observa-se que o maior impacto para FCT veio da contribuição do FCL, pois no campo das diferenças de $\theta$ os menores valores foram observados na ausência dos FCS. A máxima diferença de $\theta$ com $36 \mathrm{~h}$ de simulação foi de $7 \mathrm{~K}$ na parte nordeste do ciclone devido a ausência dos FCL e de apenas $1 \mathrm{~K}$ devido a ausência dos FCS (Figuras 9b e 9d, respectivamente). No caso do FCL, a máxima diferença de $\theta$ foi observada na região nordeste do ciclone, diferentemente do observado para o FCT (Figuras 8c e 9b). A ausência dos FCS gerou diferenças negativas de $\theta$ na maior parte do domínio nas primeiras 24 h de simulação (Figura 9c). Na costa do sul do Brasil, Uruguai e Argentina, as diferenças de temperatura associadas à ausência de FCS foram sempre negativas. No fim da simulação, a leste do centro do ciclone, os efeitos da ausência dos FCL e FCS se somam para gerar o máximo de diferença de $\theta$ associado à ausência dos FCT (Figuras $8 \mathrm{c}, 9 \mathrm{~b}, \mathrm{~d}$ ).

Para verificar qual é a extensão vertical na atmosfera dos efeitos dos FCT, analisou-se a seção vertical (orientada como indicado na Figura 3a) da diferença de $\theta$ entre o EXP CON e o EXP SFCT (Figura 10). Sobre o oceano, as diferenças positivas de $\theta$ surgem com $6 \mathrm{~h}$ de simulação nas camadas mais baixas da troposfera (Figura 10a). O impacto sobre o campo
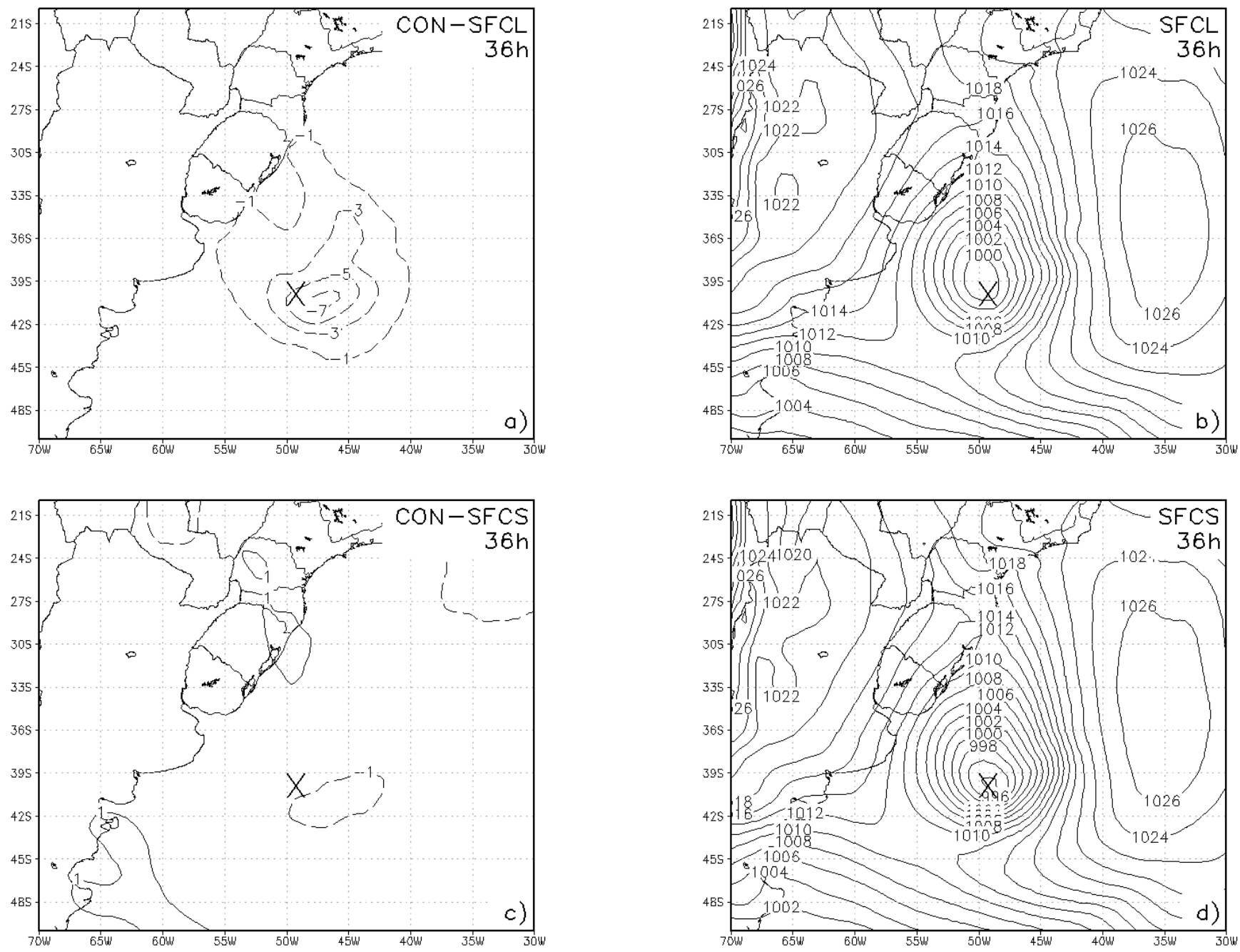

Figura 7 - Diferença de PNMM (hPa, em intervalos de $2 \mathrm{hPa}$ ) entre o EXP CON e EXP SFCL (acima) e entre o EXP CON e EXP SFCS (a baixo) para as $36 \mathrm{~h}(\mathrm{a}, \mathrm{c})$ de simulação. PNMM (hPa, em intervalos de $2 \mathrm{hPa}$ ) para as $36 \mathrm{~h}$ de simulação no EXP SFCL (b) e para o EXP SFCS (d). 
de $\theta$ aumenta em magnitude, com um máximo de $10 \mathrm{~K}$ em $950 \mathrm{hPa}$, e se estende para níveis mais altos, atingindo o nível de $680 \mathrm{hPa}$ após $30 \mathrm{~h}$ de simulação (Figuras 10b-f). Sobre o continente, as diferenças no campo de $\theta$ surgem após $18 \mathrm{~h}$ de simulação (Figura 10c), se mantêm nas camadas abaixo de 950 hPa e atingem um máximo de $7 \mathrm{~K}$ no fim da simulação (Figura 10f). Esta diferença observada entre o comportamento de $\theta$ sobre o oceano e sobre o continente reflete, em parte, a atuação do campo de movimento vertical (figura não mostrada), que pode ser deduzido do campo de PNMM e z em 500 hPa (Figura 2). Sobre o continente têm-se movimentos descendentes associados à borda do anticiclone transiente sobre a Argentina e sobre o oceano têm-se movimentos ascendentes associados ao ciclone.
Ou seja, devido ao campo de movimento vertical, o efeito dos FCT em superfície tende a ser confinados na camada inferior da troposfera sobre o continente e espalhados verticalmente sobre o oceano. Além disso, a maior diferença de LCL associada à precipitação ocorreu principalmente sobre o oceano e, sendo este um processo de grande impacto na distribuição de temperatura, ajuda a explicar porque as diferenças de $\theta$ se estenderam até níveis mais altos sobre o oceano. Desta forma, sem os FCT em superfície, a camada troposférica mais próxima à superfície oceânica fica mais fria e mais estável.

Separando-se os efeitos dos FCL e FCS, pode-se notar que o primeiro apresenta impactos maiores e são melhores distribuídos verticalmente (Figura 11). No fim da simulação, a
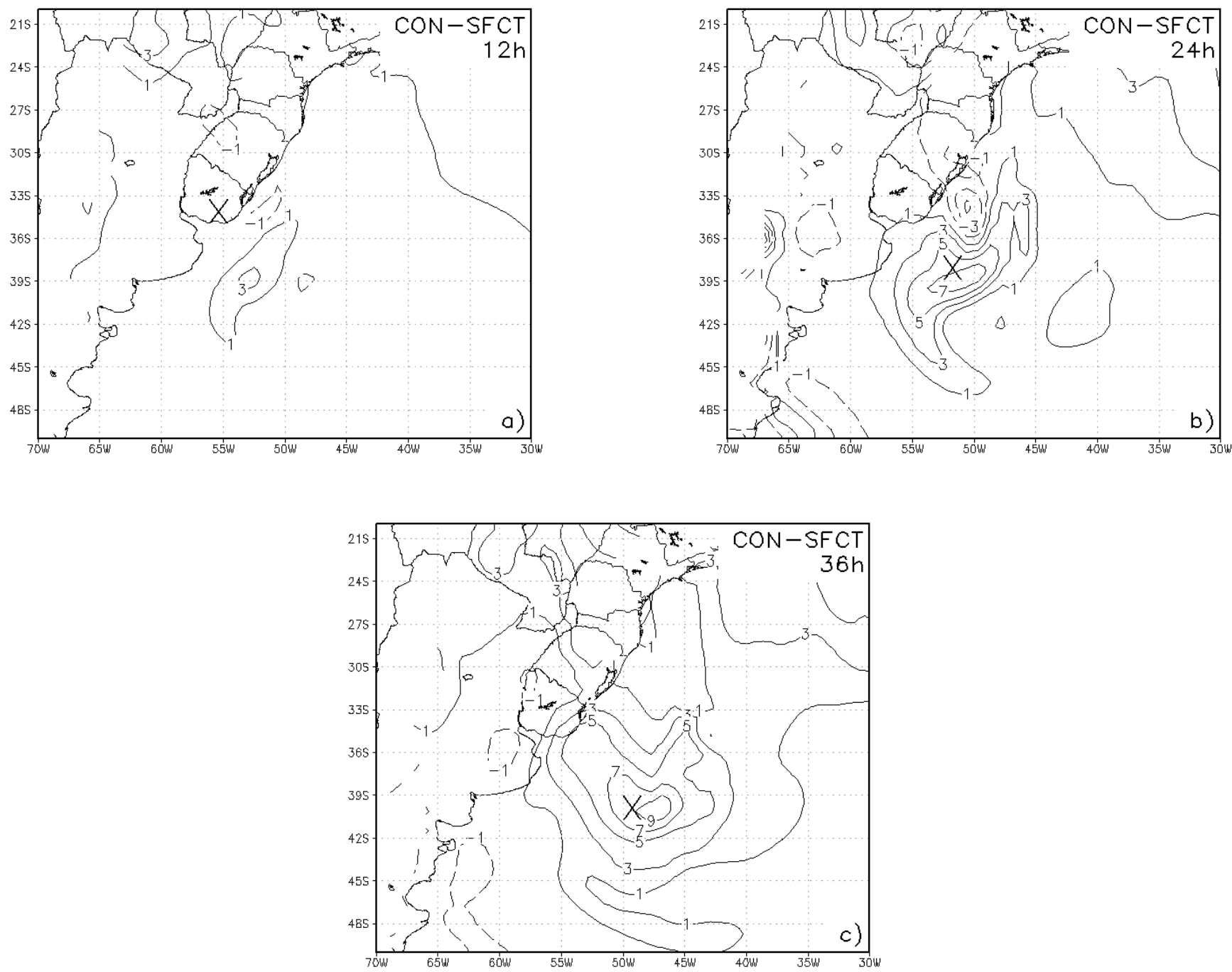

Figura 8 - Diferença de temperatura potencial (K, em intervalos de $2 \mathrm{~K}$ ) no nível de $950 \mathrm{hPa}$ entre o EXP CON e EXP SFCT para as 12 h (a), 24 h (b) e 36 h (c) de simulação. 

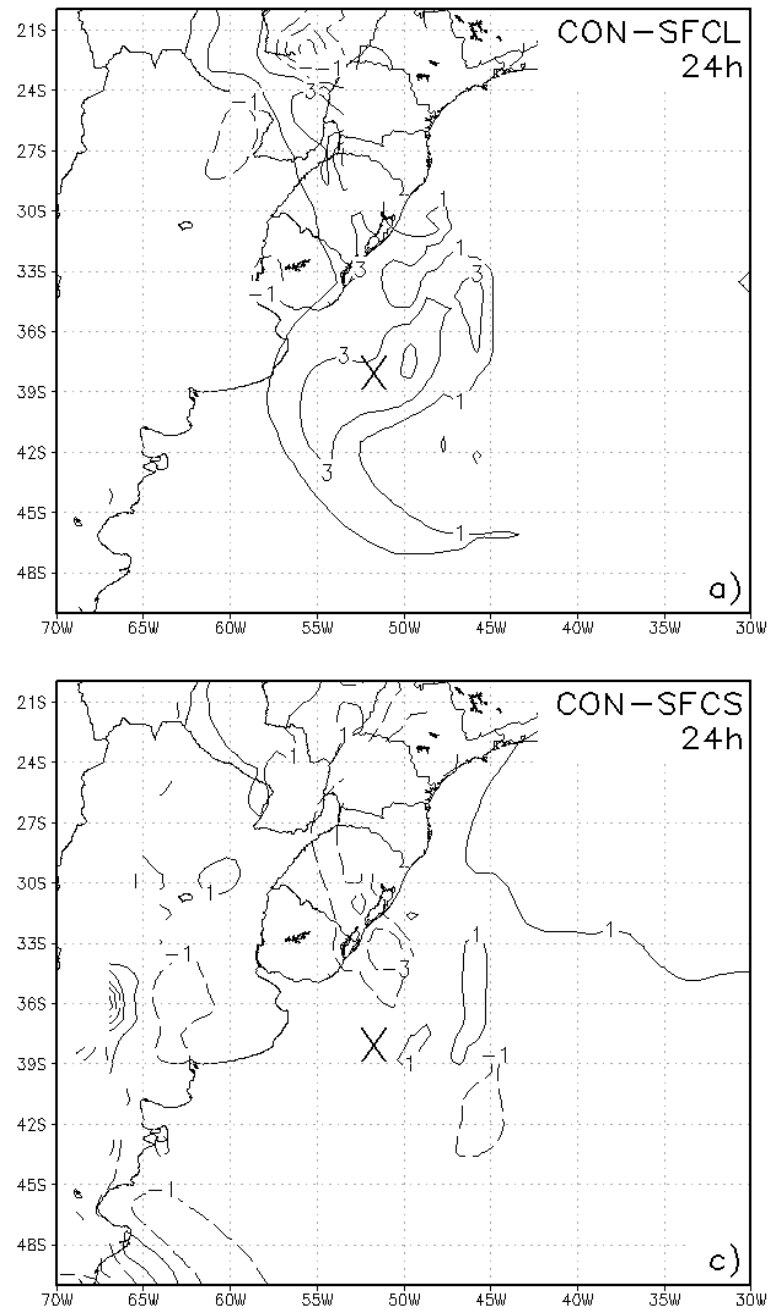
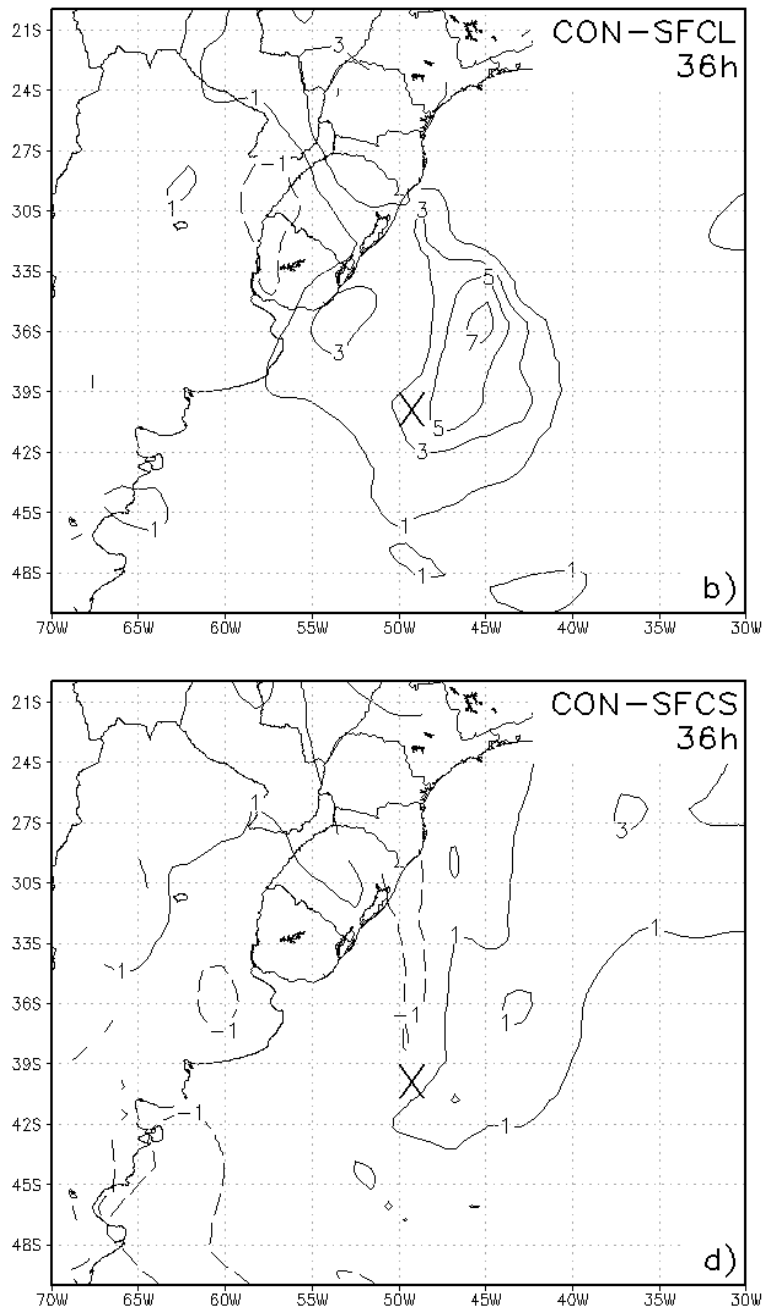

Figura 9 - Diferença de temperatura potencial (K em intervalos de $2 \mathrm{~K}$ ) em $950 \mathrm{hPa}$ entre o EXP CON e SFCL (acima) e entre o EXP CON e SFCS (a baixo) para as $24 \mathrm{~h}(\mathrm{a}, \mathrm{c})$ e $36 \mathrm{~h}(\mathrm{~b}, \mathrm{~d})$ de simulação.

ausência dos FCL produziu diferenças de $6 \mathrm{~K}$ sobre o continente (até $970 \mathrm{hPa}$ ) e oceano (entre $950 \mathrm{hPa}$ e $880 \mathrm{hPa}$ ), mas sobre a região oceânica estas diferenças foram observadas até $600 \mathrm{hPa}$ (Figura 11c). No caso do FCS as diferenças de $\theta$ atingiram no máximo $2 \mathrm{~K}$ (Figura 11f), sendo que sobre o continente e oceano próximo ao continente, as diferenças de $\theta$ foram negativas como era de se esperar devido à ocorrência de FCS negativos (Figura 5). Ressalta-se que os impactos dos FCL e FCS somados foram inferiores aos impactos devido aos FCT. Embora este último seja a soma dos dois primeiros, a não-linearidade e a interdependência dos processos envolvidos não garantem que os efeitos da ausência dos FCT sejam iguais à soma dos efeitos da ausência dos FCL e FCS obtidos separadamente.

Os efeitos da ausência dos FCT no campo de umidade específica em $950 \mathrm{hPa}$ podem ser vistos na Figura 12. Nas primeiras $12 \mathrm{~h}$ de simulação, as maiores diferenças de umidade específica entre o EXP CON e o EXP SFCT foram observadas sobre o continente na região centro-oeste do Brasil (Figura 12a). Neste período, a costa brasileira e do Uruguai registraram diferenças de $1 \mathrm{gkg}^{-1}$, com máximo de $3 \mathrm{gkg}^{-1}$ (Figura 12a), indicando que a atmosfera nessa região se tornou mais seca no EXP SFCT do que no EXP CON. A área que apresentou maior diferença de umidade específica encontra-se sobre o Oceano Atlântico ao norte de $30^{\circ} \mathrm{S}$ entre $35^{\circ} \mathrm{W}$ e $45^{\circ} \mathrm{W}$ (Figuras 12a-c), correspondendo aproximadamente à região em que ocorreram os maiores FCT (Figura 3). Próximo ao ciclone, as diferenças de umidade específica aumentaram quando o sistema se deslocou para o oceano, atingindo valores máximos de $3 \mathrm{gkg}^{-1}$ no centro do ciclone com $24 \mathrm{~h}$ de simulação (Figura 12b) e $5 \mathrm{gkg}^{-1}$ a leste do centro do ciclone com $36 \mathrm{~h}$ de simulação (Figura 12c).

Analisando-se separadamente a ausência dos FCL e FCS (Figura 13), nota-se que os FCL apresentaram maiores efeitos 

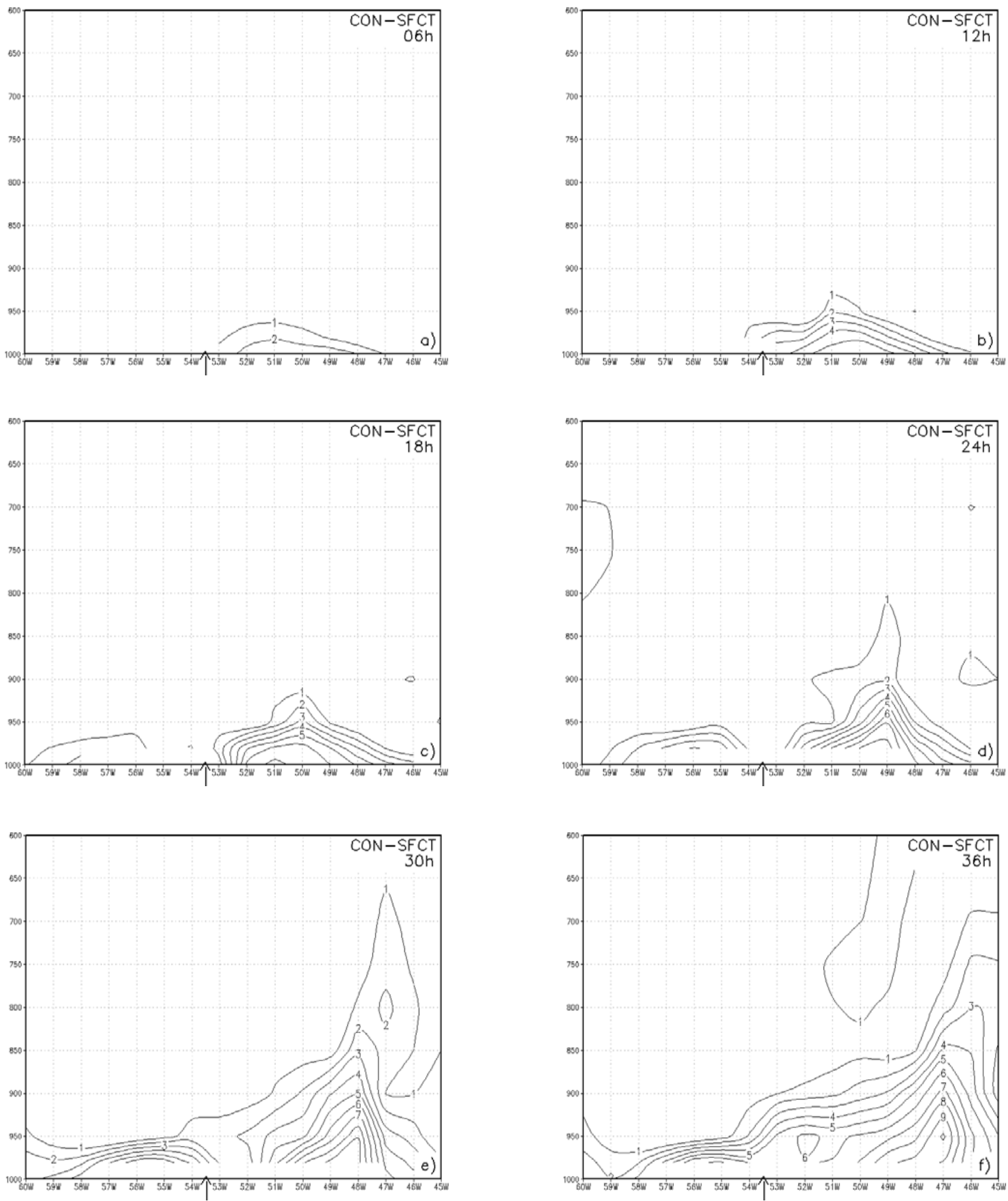

Figura 10 - Seção vertical da diferença de temperatura potencial (K, em intervalos de $1 \mathrm{~K}$ ) entre o EXP CON e SFCT para as $06 \mathrm{~h}$ (a), $12 \mathrm{~h}(\mathrm{~b}), 18 \mathrm{~h}$ (c), 24 h (d), 30 h (e) e 36 h (f) de simulação. A seta na parte inferior das figuras indica o limite entre continente (a esquerda) e oceano (a direita). 

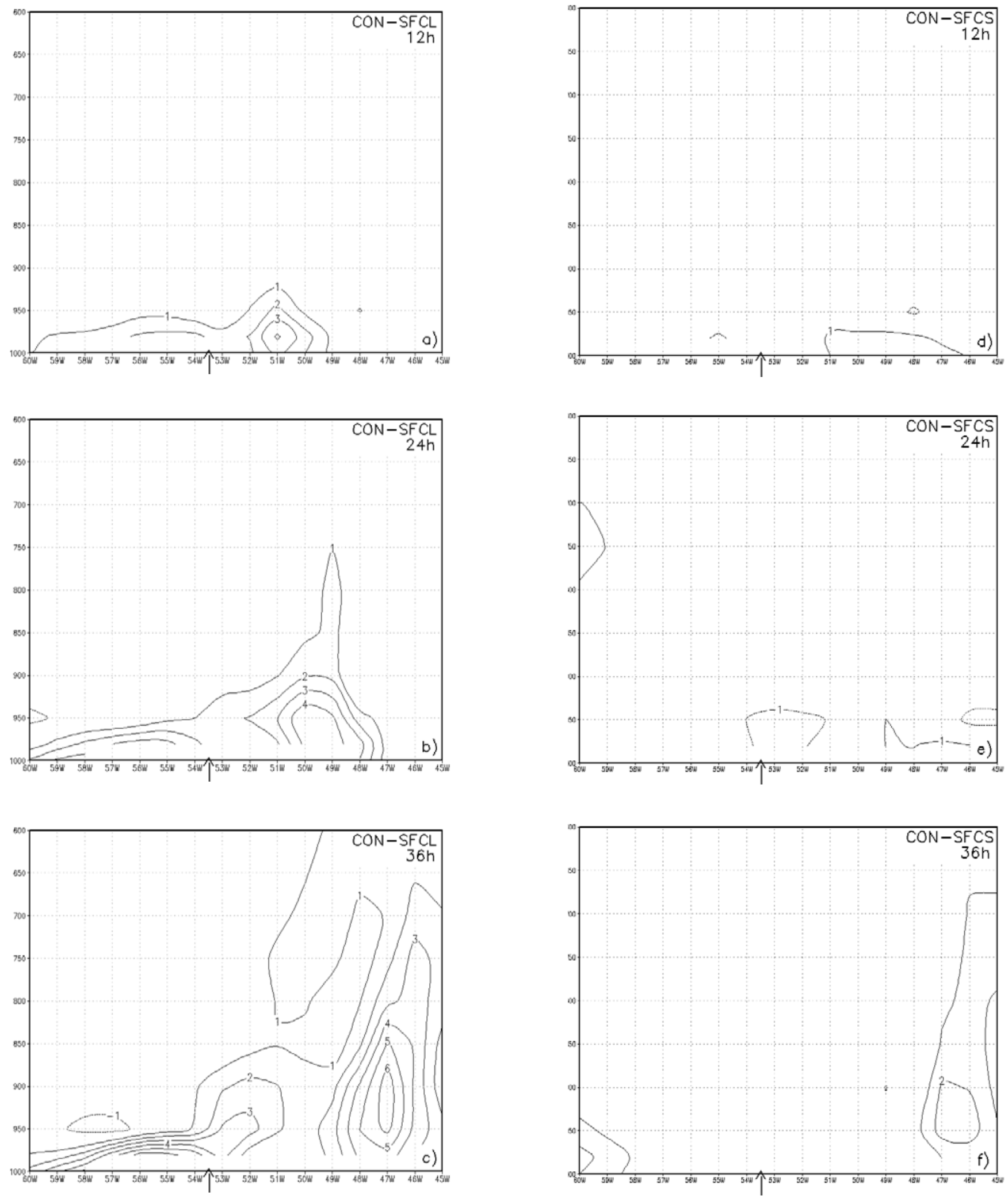

Figura 11 - Seção vertical da diferença de temperatura potencial (K, em intervalos de $1 \mathrm{~K}$ ) entre o EXP CON e SFCL (a esquerda) e entre o EXP CON e SFCS (a direita) para as $12 \mathrm{~h}(\mathrm{a}, \mathrm{d}), 24 \mathrm{~h}$ (b, e) e $36 \mathrm{~h}$ (c, f) de simulação. A seta na parte inferior das figuras indica o limite entre continente (a esquerda) e oceano (a direita). 

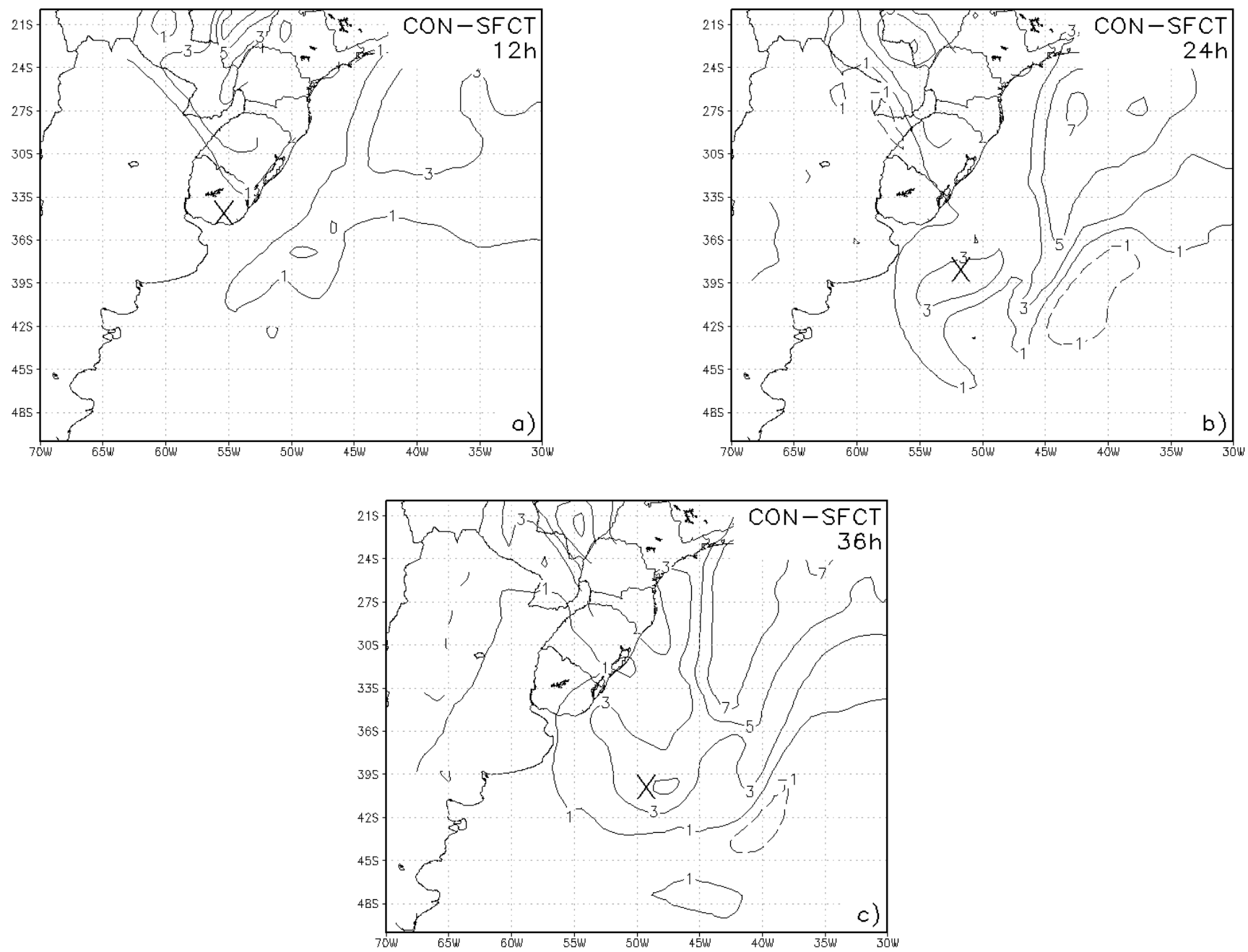

Figura 12 - Como na Figura 8, mas para a umidade específica $\left(\mathrm{gkg}^{-1}\right)$.

no campo de umidade específica em $950 \mathrm{hPa}$ do que os FCS. Este resultado era esperado, pois, os FCL representam o fluxo vertical de umidade, porém houve regiões em que o maior impacto foi devido aos FCS, como por exemplo, próximo a $44^{\circ} \mathrm{W}$ e $35^{\circ} \mathrm{S}$ após $24 \mathrm{~h}$ de simulação (Figura 13c). Em parte este resultado pode ser associado ao fato que a ausência dos FCS (que na região em questão era positivo, ver Figuras $5 b$ e 5c) gerou um ambiente mais frio (Figura 9c) e, como se sabe, ar mais frio tem menor capacidade de reter umidade do que ar mais quente. Assim, a ausência dos FCS através do não aquecimento da camada em contato com o oceano pode diminuir o FCL e gerar um ambiente mais seco. Isto explicaria o máximo de diferença de umidade específica da ordem de $5 \mathrm{~g}$ $\mathrm{kg}^{-1}$, observado sobre o Oceano Atlântico após $24 \mathrm{~h}$ de simulação (Figura 13c). Interessante notar também, que as diferenças de umidade específica aumentaram pouco entre as $24 \mathrm{~h}$ e as 36 $\mathrm{h}$ de simulação, isto porque nos EXP sem fluxos de calor em superfície, a quantidade de umidade específica na atmosfera foi menor do que no EXP CON, mas com o aumento do tempo de simulação, a formação da precipitação também foi reduzida, deixando mais vapor d'água na atmosfera e contrabalançando a ausência de fluxo de umidade proveniente da superfície oceânica.

Com relação à seção vertical da diferença de umidade específica (Figura 14), verifica-se comportamento similar ao observado no caso da $\theta$ (Figura 10), ou seja, valores predominantemente positivos, com magnitudes maiores na camada inferior da troposfera próximo à superfície oceânica, distribuindo-se verticalmente com o aumento do tempo de simulação. Chama-se a atenção ao fato de que as diferenças de umidade específica foram demasiadamente restritas aos baixos níveis da troposfera. Uma explicação para tal comportamento, deve-se ao fato que no EXP CON tem-se 
uma fonte de umidade em baixos níveis (superfície oceânica) e um sumidouro (precipitação) em médios e altos níveis, enquanto no EXP SFCT e no EXP SFCL desligou-se a fonte de umidade (FCL ausente), e o sumidouro (precipitação) se tornou menos eficiente, pois como será mostrado adiante, a precipitação diminuiu. Além disso, deve-se levar em conta também, que os movimentos verticais ascendentes sobre a região transportam umidade dos baixos para os médios e altos níveis. O balanço de tais processos indica que em médios e altos níveis a quantidade de umidade sofreu pouca alteração com a ausência dos FCT, mas em baixos níveis, a ausência da fonte de umidade e a presença de movimentos verticais ascendentes, fizeram com que a ausência dos FCT fosse mais visível.
Analisando-se os efeitos dos FCL e FCS separadamente, pode-se notar que o maior impacto na umidade específica foi devido aos FCL (Figura 15). Chama a atenção o fato de que com $12 \mathrm{~h}$ de simulação sem FCL, a diferença de umidade específica atinge o máximo de $3 \mathrm{gkg}^{-1}$ e se mantêm neste valor até o fim da simulação (Figuras 15a-c). Tal comportamento é resultado do balanço parcial entre a ausência da fonte de umidade na base do modelo (FCL ausente) e da diminuição do sumidouro de umidade em médios e altos níveis (associado a redução na precipitação), como se pode ver pela diferença negativa observada em $50^{\circ} \mathrm{W}$ entre $800 \mathrm{hPa}$ e $750 \mathrm{hPa}$, após $36 \mathrm{~h}$ de simulação (Figura 15c). Outra característica interessante é que na ausência dos FCS, as diferenças de umidade específica foram máximas em 950
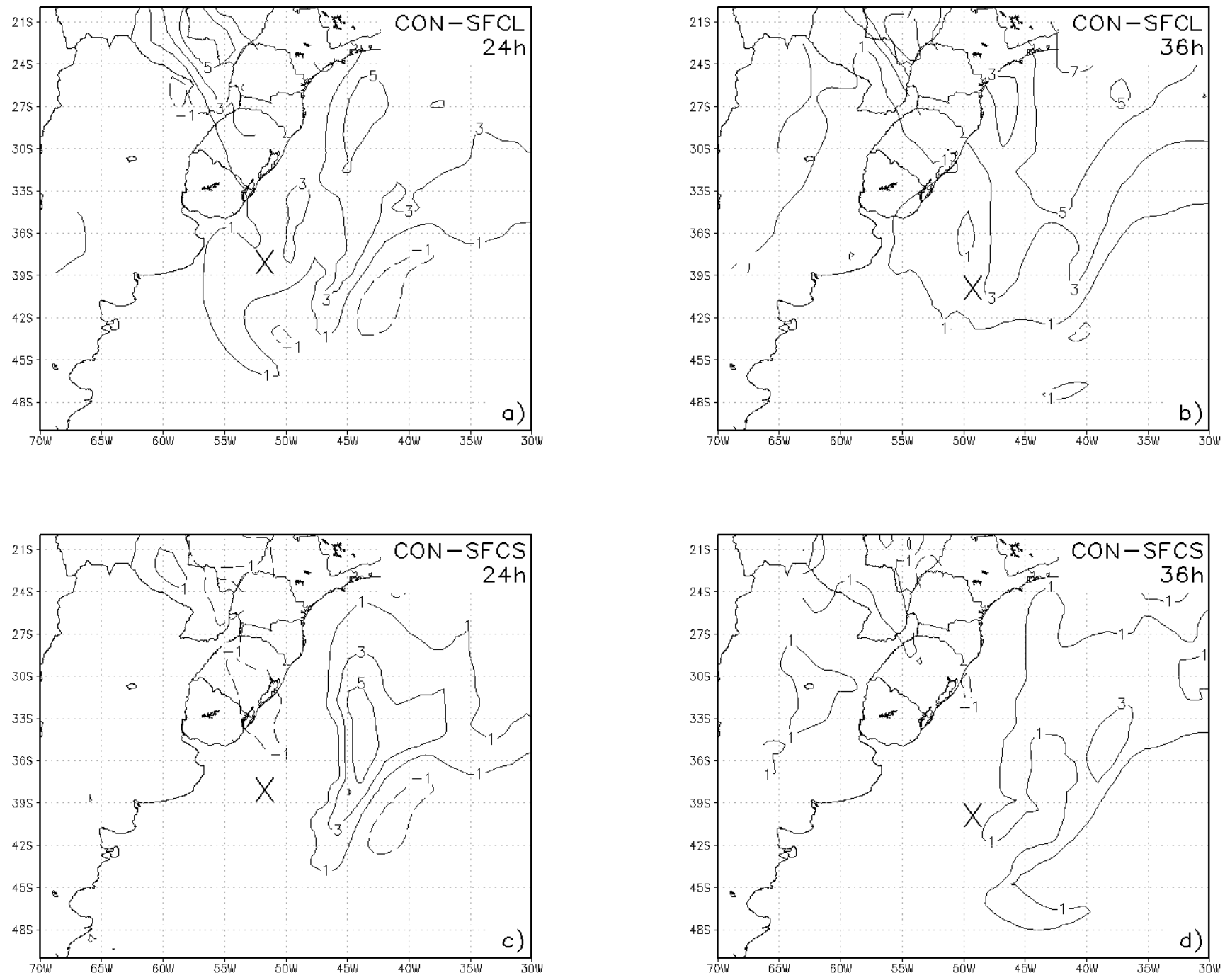

Figura 13 - Como na Figura 9, mas para a umidade específica $\left(\mathrm{gkg}^{-1}\right)$. 

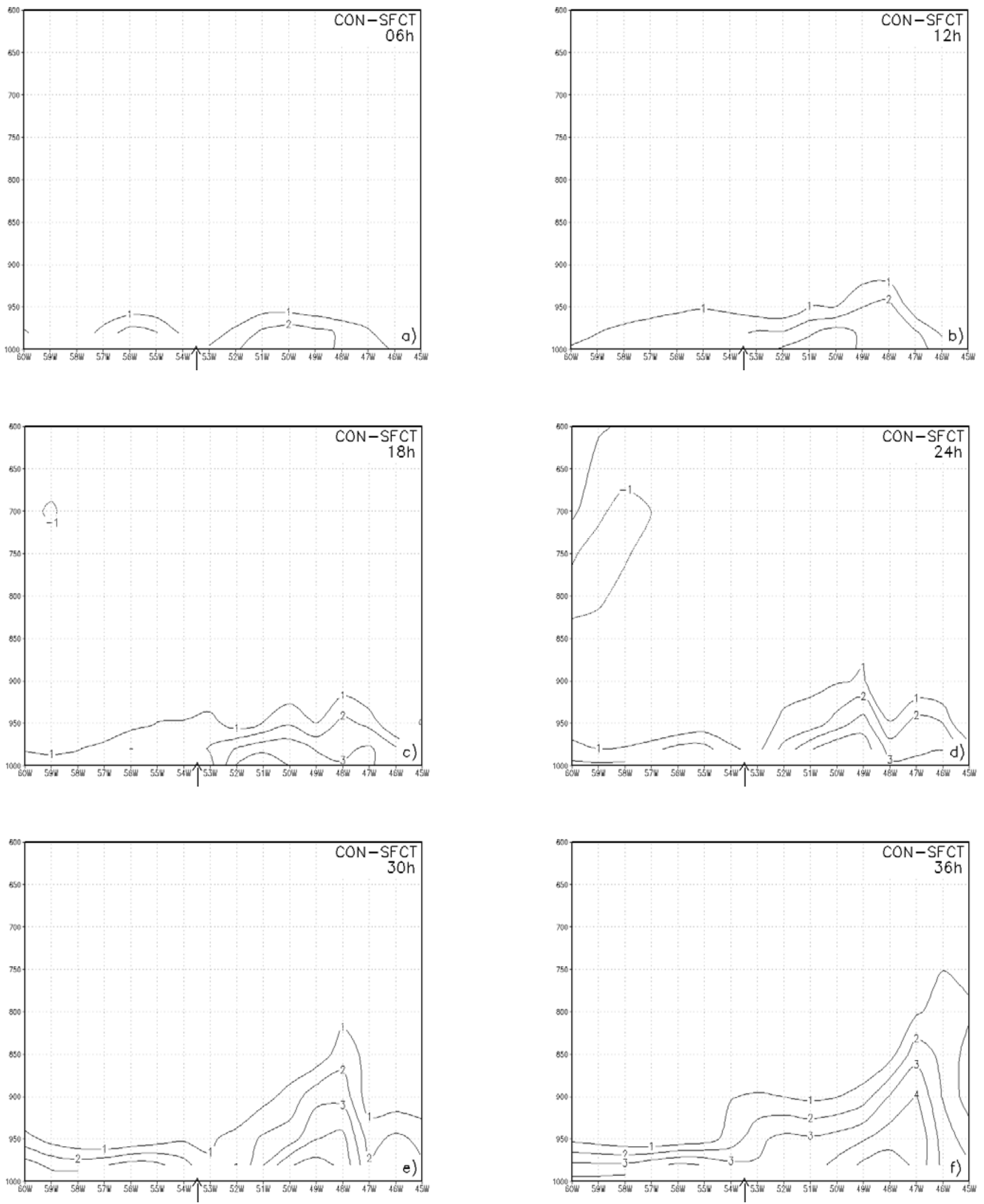

Figura 14 - Como na Figura 10, mas para a umidade específica $\left(\mathrm{gkg}^{-1}\right)$. 

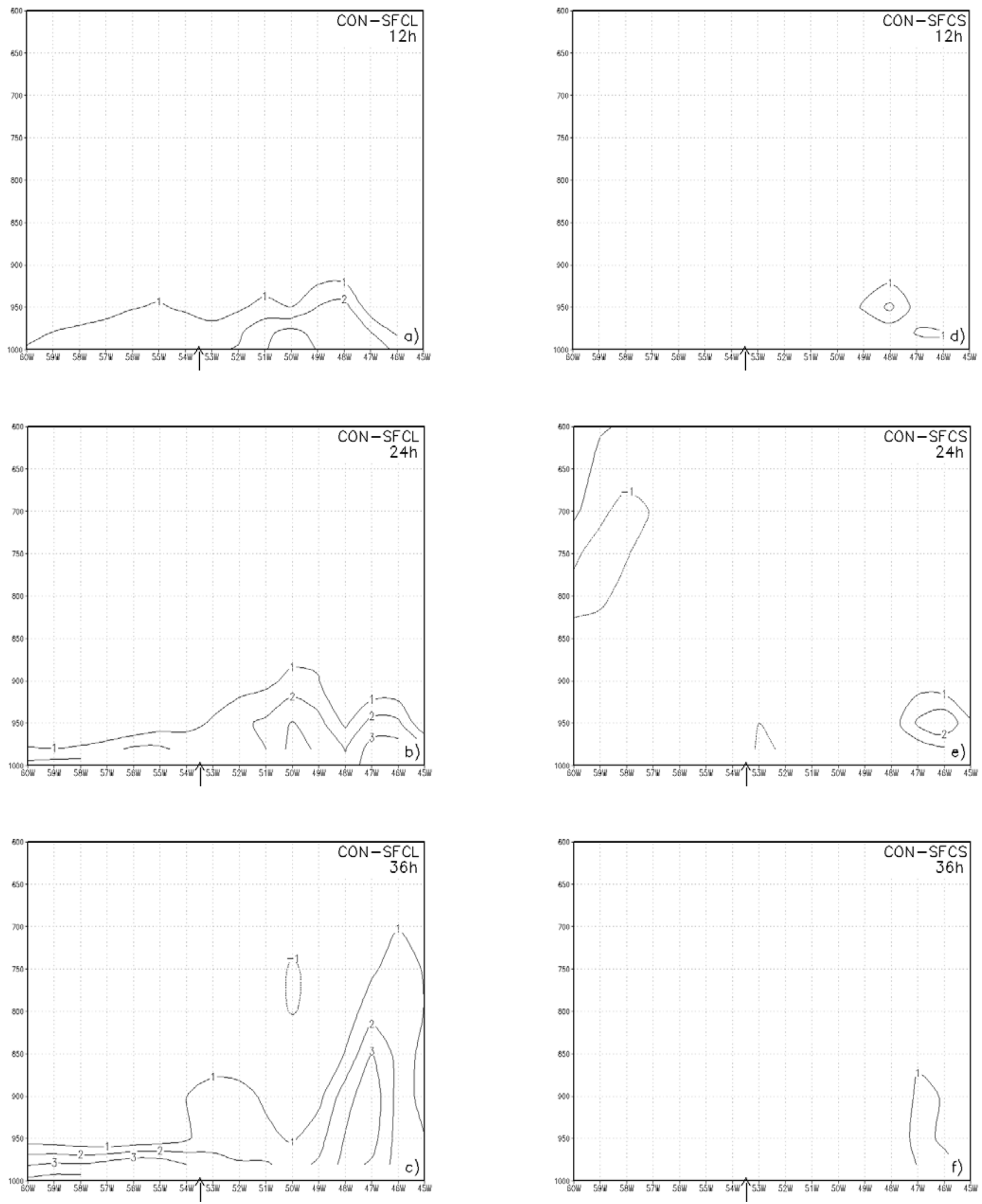

Figura 15 - Como na Figura 11, mas para a umidade específica $\left(\mathrm{gkg}^{-1}\right)$ 

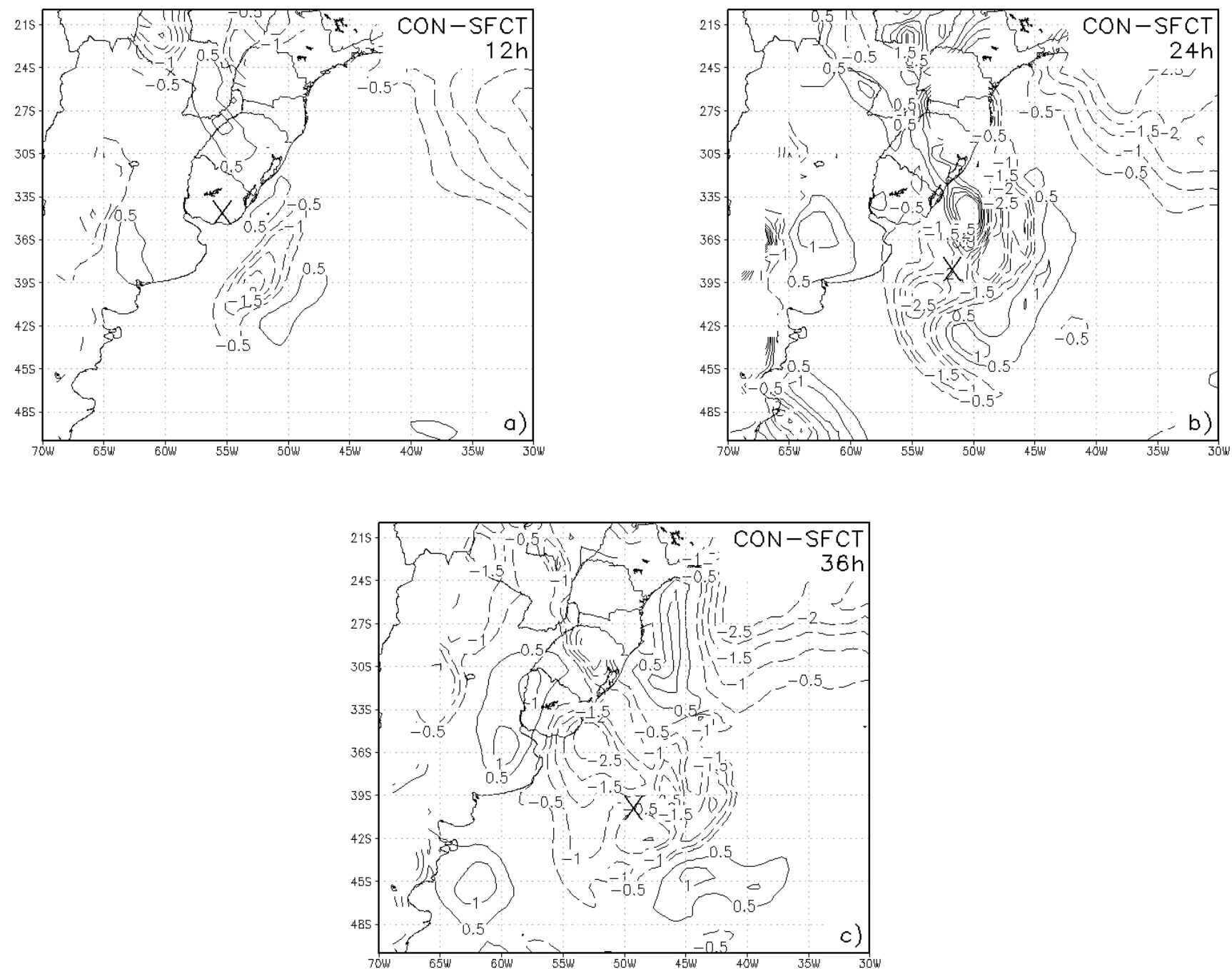

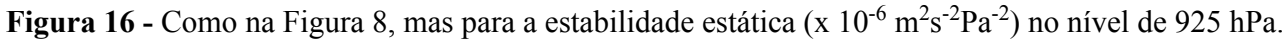

hPa (Figuras 15d-f), diferentemente da ausência dos FCL, que geraram diferenças maiores próximas a superfície oceânica.

Devido ao fato de que os FCT forneceram calor e umidade para a atmosfera, principalmente para a troposfera inferior sobre o oceano, a troposfera tornou-se mais estável na ausência dos FCT, como pode ser visto na Figura 16. As diferenças no campo de estabilidade estática entre os EXP CON e EXP SFCT mostram em geral, valores negativos, indicando que a estabilidade estática foi maior no EXP SFCT do que no EXP CON. Grande parte das diferenças positivas na diferença de estabilidade estática está associada aos FCS negativos, como pode ser notado comparando-se as Figuras 5 e 16. Após $12 \mathrm{~h}$ de simulação (Figura 16a) nota-se que a ausência dos FCT torna a camada limite planetária sobre o oceano mais estável, exceto uma pequena área próxima à costa do Rio Grande do Sul, indicando um efeito pré-condicionador dos FCT como sugerido por alguns estudos (Bosart e Lin, 1984; Kuo e Low-Nam, 1990; Kuo et al., 1991a, b). Este papel de pré-condicionar o ambiente, favorecendo o rápido desenvolvimento do ciclone, indica que os FCT são importantes antes do período de mais rápido desenvolvimento do ciclone, ou seja, durante as primeiras $12 \mathrm{~h}$ de simulação (Figura 2).

Uma análise mais detalhada dos efeitos dos FCT na estabilidade térmica, pode ser feita através da sequência temporal da secção vertical da diferença da estabilidade estática entre os EXP CON e EXP SFCT (Figura 17). Após 6h de simulação observa-se que as diferenças negativas surgem sobre o oceano na camada mais inferior do modelo (Figura 17a), aumentando em magnitude e distribuindo-se verticalmente com o decorrer da simulação (Figuras 17b-f). O máximo de 

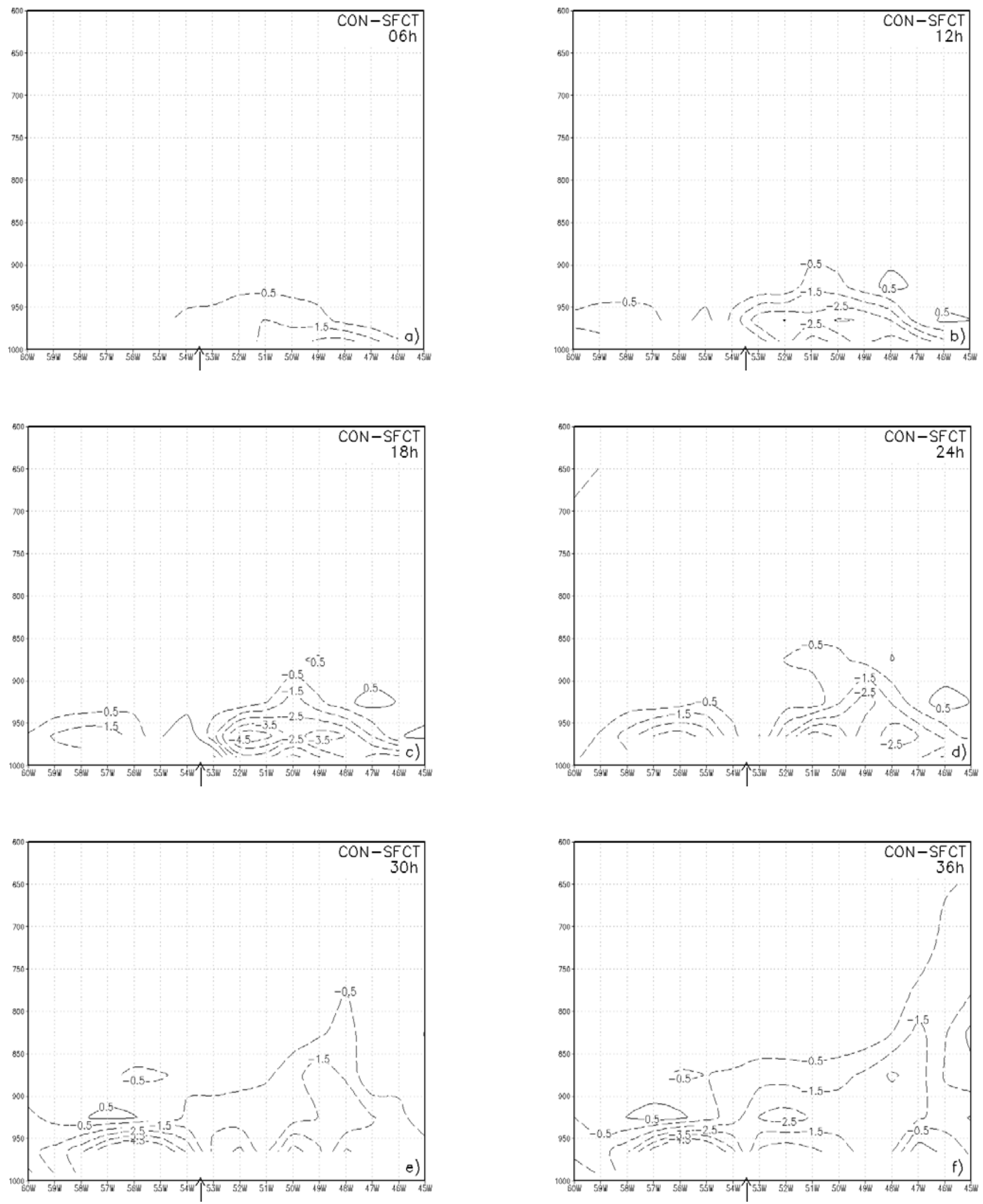

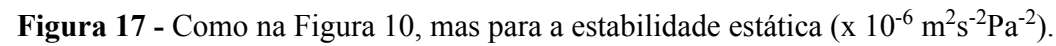



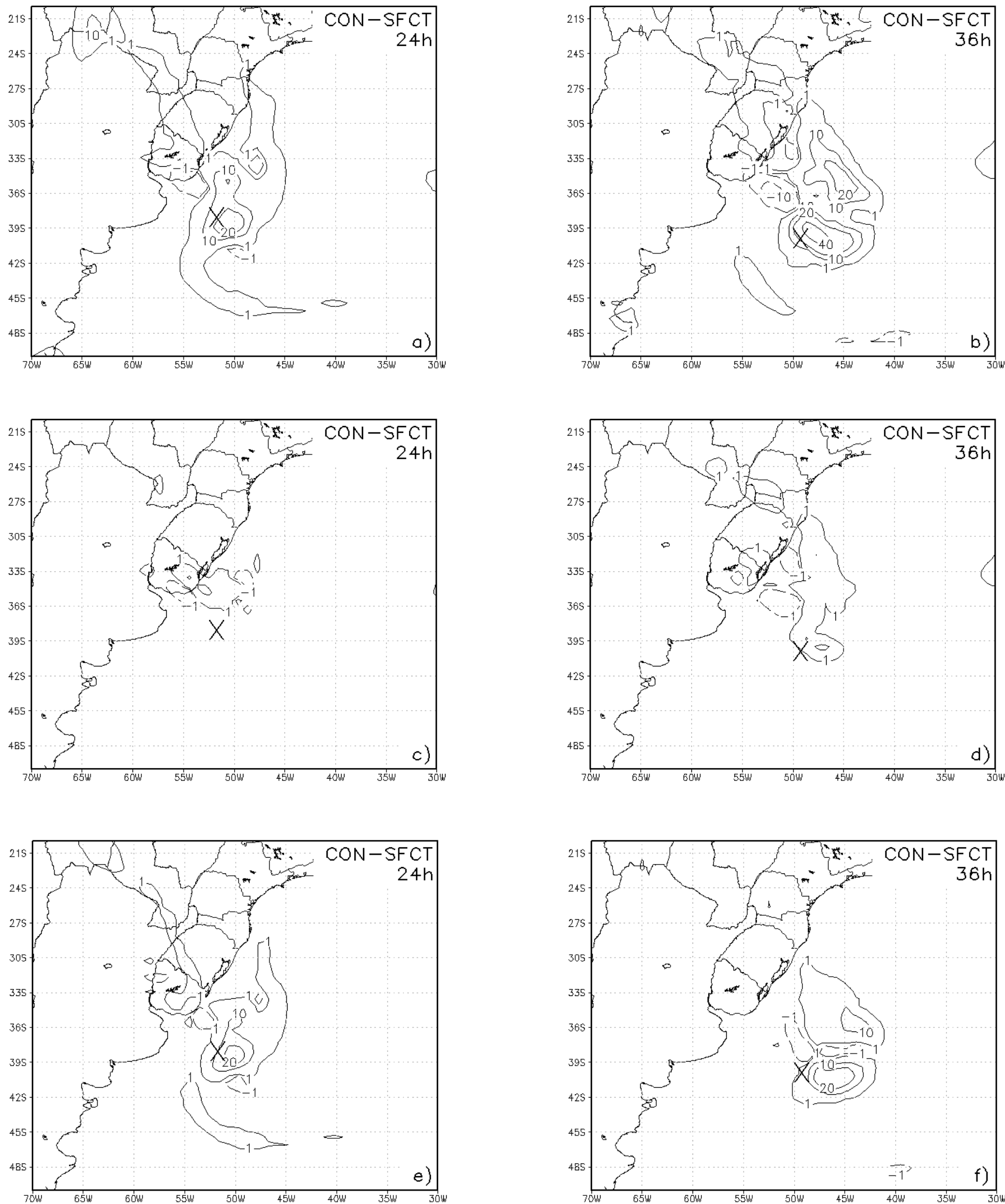

Figura 18 - Diferença de PAT (a, b), PAC (c, d) e PAGE (e, f) entre o EXP CON e SFCT, para as 24 h (a esquerda) e 36 h (a direita) de simulação. Contornos de $-40,-20,-10,-1,1,10,20,40 \mathrm{~mm} / 12 \mathrm{~h}$. 
diferença de estabilidade estática da ordem de $-4,5 \times 10^{-6} \mathrm{~m}^{2}$ $\mathrm{s}^{-2} \mathrm{~Pa}^{-2}$, foi observado sobre o oceano com apenas $18 \mathrm{~h}$ de simulação (Figura 17c), confirmando o efeito pré-condicionador dos FCT. Sobre o continente, o máximo (de mesmo valor) da diferença da estabilidade térmica foi observado entre $30 \mathrm{~h}$ e 36 h de simulação (Figuras 17e e 17f). Portanto, a ausência dos FCT gera um ambiente mais estável, principalmente nas camadas inferiores da troposfera sobre o oceano no período anterior ao estágio de mais rápido desenvolvimento do ciclone.

A ausência dos FCT em superfície provocou alterações consideráveis no campo de precipitação como pode ser visto na Figura 18, a qual apresenta os campos de diferença de PAT (Figuras 18a,b), PAC (Figura 18c,d) e PAGE (Figuras 18e,f) entre o EXP CON e o EXP SFCT. As diferenças de PAT foram, em sua maioria positivas (menor precipitação no EXP SFCT do que no EXP CON) e localizadas no setor quente do ciclone, principalmente ao longo da frente quente (Figura 18a-b). O máximo da diferença de PAT foi superior a $40 \mathrm{~mm} / 12 \mathrm{~h}$ para o EXP SFCT (Figura 18b), $50 \mathrm{~mm} / 12 \mathrm{~h}$ para o EXP SFCL (figura não mostrada) e de $20 \mathrm{~mm} / 12 \mathrm{~h}$ para o EXP SFCS (figura não mostrada). A física e os métodos numéricos utilizados no modelo ajudam a entender porque a diferença de PAT foi maior no EXP SFCL do que no EXP SFCT. No EXP SFCL não houve fornecimento de umidade da superfície para a atmosfera, mas os FCS permaneceram aquecendo as camadas mais baixas da troposfera. Nesta situação, o nível de supersaturação e, por consequência, a geração de PAGE, foi menor do que no EXP SFCT, onde não houve aquecimento e fornecimento de umidade nos baixos níveis da troposfera. Isso explica porque houve menor PAT no EXP SFCL do que no EXP SFCT. Houve também, uma pequena área de diferenças negativas de precipitação PAT a noroeste do centro do ciclone, que se deve ao fato de que esta região foi caracterizada por FCS negativos (Figura 5c-d) e um mínimo de FCL (Figura 4c-d) e FCT (Figura 3c-d). Em áreas com este padrão de FCL e FCS em superfície, a estabilidade para movimentos verticais ascendentes é maior do que em áreas com FCS positivos e com fortes FCL, resultando em menor formação de PAT. Analisando-se separadamente os campos de PAC e PAGE, pode-se notar que a maior parte da diferença na precipitação foi devido a PAGE (Figura 18e-f), com a PAC sendo menos afetada (Figura $18 \mathrm{c}-\mathrm{d}$ ). Isto sugere que a condição de instabilidade térmica (uma das condições para geração de PAC) foi afetada em menor grau do que a supersaturação do ambiente (condição para geração de PAGE). Assim, na ausência do FCT, ocorre redução da precipitação associada à ciclogênese, principalmente na precipitação resolvida na grade.

A evolução do campo de FCT, mostrando valores elevados antes do período de máximo de desenvolvimento, e o impacto da ausência dos FCT nos campos de temperatura e umidade específica, sugerem que os FCT sejam importantes antes do período de mais rápido desenvolvimento do sistema. Porém devido ao tempo de resposta, os seus efeitos (ambiente termicamente mais instável, maior conteúdo de umidade e maior liberação de calor latente) continuam sendo importantes durante o período de máximo desenvolvimento.

\section{CONCLUSÕES}

Este artigo abordou os efeitos dos fluxos de calor latente e sensível em superfície no desenvolvimento de um ciclone sobre a costa leste da América do Sul, entre os dias 24 e 26 de julho de 1998. Isto foi verificado através de simulações com o modelo regional MAL-JAPA, o qual mostrou uma taxa de intensificação de $16 \mathrm{hPa} / 24 \mathrm{~h}$, o que representa um valor ligeiramente abaixo daquele que o classificaria como uma ciclogênese explosiva (da ordem de $16,3 \mathrm{hPa} / 24 \mathrm{~h}$ ), segundo o critério de Sanders e Gyakum (1980). A taxa de intensificação foi reduzida para $10 \mathrm{hPa} / 24 \mathrm{~h}$ nas simulações sem fluxos de calor total e latente em superfície, e não sofreu alteração na simulação sem fluxo de calor sensível em superfície.

As diferenças nos campos meteorológicos devido à ausência dos FCT em superfície aumentaram com o tempo, tanto em magnitude quanto em área. Este comportamento se deve a dois fatores: 1) Um processo cumulativo no tempo, pois a ausência dos FCT em superfície vai gerando perturbações no campo de temperatura (FCS) e de umidade (FCL), que aumentam com o tempo de simulação; 2) Uma reação em cadeia em que outros processos físicos e campos meteorológicos vão sofrendo alterações, como por exemplo, a velocidade do vento, a flutuabilidade do ar, a liberação de calor latente e outros.

Os FCT em superfície geram uma camada na troposfera inferior mais quente e mais úmida do que no caso sem os fluxos. Em uma estratificação como esta, a troposfera apresenta alto nível de instabilidade potencial, ou seja, ela apresenta instabilidade que poderá ser liberada durante um levantamento forçado. No caso estudado, o levantamento forçado foi realizado pelo avanço das frentes frias e quentes associadas à ciclogênese em superfície. Em decorrência do levantamento forçado, ocorre a liberação de calor latente, que passa a ser o mecanismo diabático dominante. No EXP SFCT (com argumentos similares para os EXPs SFCL e SFCS), a camada inferior da troposfera fica mais fria e mais seca e, portanto, há menos instabilidade potencial, e a ciclogênese tende a ser menos intensa. Ressalta-se que a ciclogênese ocorre com ou sem a presença de FCT em superfície e isto se deve ao fato de que a instabilidade baroclínica é o mecanismo dominante no desenvolvimento da ciclogênese extratropical na região de estudo como sugerido por Gan e Rao (1991). Assim, os FCT em superfície e outros processos influenciados por esses fluxos (tal como a liberação de calor latente), são mecanismos 
que tornam a ciclogênese mais intensa (ou explosiva), mas não são responsáveis pela ciclogênese propriamente dita.

Ressalta-se que os fluxos em superfície têm o papel de pré-condicionar o ambiente, tornando-o favorável ao desenvolvimento mais intenso do ciclone extratropical. Embora os fluxos sejam importantes antes do período de mais rápido desenvolvimento do ciclone, os seus efeitos são sentidos durante este período e não somente na fase inicial do seu ciclo de vida, devido ao tempo de resposta que a atmosfera leva para responder à retirada ou inclusão de calor pelos fluxos na superfície. $\mathrm{Na}$ fase de mais rápido desenvolvimento, os efeitos dos fluxos de calor em superfície são sentidos através da menor estabilidade térmica e do maior conteúdo de umidade, os quais levam a uma intensificação da circulação vertical e da liberação de calor latente.

Conclui-se que o Oceano Atlântico Sul próximo à costa do sul do Brasil e Uruguai, influenciou de forma decisiva a intensidade do ciclone estudado e, que provavelmente, apresente forte influência na maioria das ciclogêneses que ocorrem na costa leste da América do Sul. Desta forma, é preciso atenção em relação aos fluxos de calor em superfície parametrizados pelos modelos utilizados em pesquisas e previsão operacional nos países da costa leste do Oceano Atlântico Sul.

\section{5- AGRADECIMENTOS}

O primeiro autor agradece ao Conselho Nacional de Desenvolvimento Científico e Tecnológico (CNPq) pela bolsa de mestrado. Agradecemos ao Dr. Valdir Innocentini, Dra. Rosmeri Porfírio da Rocha e ao Dr. Nivaldo Silveira Ferreira pelos esclarecimentos relativos ao modelo regional utilizado. Este estudo faz parte do projeto intitulado: "Dinâmica da ciclogênese no Atlântico Sul”, processo número 95/4573-5, financiado pela Fundação deAmparoà Pesquisa do Estado de São Paulo(FAPESP).

\section{6- REFERÊNCIAS BIBLIOGRÁFICAS}

ATLAS, R. The role of oceanic fluxes and initial data in the numerical prediction of an intensive coastal storm. Dynamics of Atmospheres and Oceans, v. 10, p. 359-388, 1987.

BLUESTEIN, H. B. Synoptic-dynamic meteorology in midlatitudes. Vol. I. New York: Oxford University Press, 1992. 431p.

BONATTI, J. P.; RAO, V. B. Moist baroclinic instability in the development of North Pacific and South-American intermediate-scale disturbances, Journal of the Atmospheric Sciences, v. 44, p. 2657-2667, 1987.

BONATTI, J. P. Análise da capacidade do modelo global operacional do CPTEC/COLA para simular a evolução de meso-escala usando alta resolução: estudo de caso. In: CONGRESSO BRASILEIRO DE METEOROLOGIA,
2000, Rio de Janeiro. Anais... Florianópolis: SBMET, 2000a. v. 1.

BONATTI, J. P. Capacidade do modelo global operacional do CPTEC/COLA T062L28 em simular a evolução de ciclogênese de meso-escala: estudo de caso. In: CONGRESSO BRASILEIRO DE METEOROLOGIA, 2000, Rio de Janeiro, 2000. Anais... Florianópolis: SBMET, 2000b, v. 1.

BOSART, L. F.; LIN, S. C. A diagnostic analysis of the Presidents' day storm of February 1979. Monthly Weather Review, v. 112, p. 2148-2177, 1984.

BUSSINGER, J. A.; WYNGAARD, J. C.; IZUMI, Y.; BRANDLEY, E. F. Flux-profile relationships in the atmospheric surface layer. Journal of the Atmospheric Sciences, v. 28, p. 181-189, 1971.

CHANG, S. W.; HOLT, T. R.; SASHEGYI, K.D. A numerical study of the ERICA IOP 4 marine cyclone. Monthly Weather Review, v. 124, p. 27-46, 1996.

CHEN, T.-C.; KUO, Y.-H.; ZHANG, P.-Z; BAI, Q.-F. Climatology of explosive cyclones off the east Asian coast. Monthly Weather Review, v. 120, p. 3029-3035, 1992.

DAVIS, C.A.; EMANUEL, K.A. Observational evidence for the influence of surface heat fluxes on rapid marine cyclogenesis. Monthly Weather Review, v. 116, p. 2649-2659, 1998.

GAN, M. A.; RAO, V. B. Surface cyclogenesis over South America. Monthly Weather Review, v. 119, p. 1293-1302, 1991.

GAN, M. A.; RAO, V. B. Case studies of cyclogenesis over South America. Meteorology Applied, v. 3, p. 359-368, 1996.

GELEYN, J. F. On a simple, parameter-free partition between moistening and precipitation in the Kuo scheme. Monthly Weather Review, v. 113, p. 405-417, 1985.

GYAKUM, J. R, CARRERA, M., ZHANG, D-L, MILLER, S. CAVEEN, J., BENOIT, R., BLACK, T., BUZZI, A. CHOUINARD, C. FANTINI, M., FOLLONI, C., KATZFEY, J. J., KUO, Y-H., LALAURETTE, F., LOWNAM, S., MAILHOT, J., MALGUZZI, P. MCGREGOR, J. L., NAKAMURA, M., TRIPOLI, G., WILSON, C. A regional model intercomparison using a case of explosive oceanic cyclogenesis. Weather and Forcasting, v. 11, p. 521-543, 1996.

HOLLAND, G. J., LYNCH, A. H.; LESLIE, L. M. Australian east-coast cyclones. Part I: Synoptic overview and case study. Monthly Weather Review, v. 115, p. 3024-3036, 1987.

HOLT, T. R.; S. W. CHANG. A numerical investigation of the effects of timing of diabatic processes in the coastal cyclogenesis of GALE IOP 2. Monthly Weather Review, v. 121, p. 1007-1029, 1993.

INNOCENTINI, V.; CAETANO NETO, E. D. A case study 
of the 9 August 1988 South Atlantic storm: Numerical simulations of the wave activity. Weather and Forecasting, v. 11, p. 78-88, 1996.

JUANG, H. -M. H.; OGURA, Y. A. A case study of rapid cyclogenesis over Canada. Part II: Simulations. Monthly Weather Review, v. 118, p. 674-704, 1990.

KALNAY, E., KANMITSU, M.; KISTLER, R.; COLLINS, W.; DEAVEN, D.; GANDIN, L.; IREDELL, M.; SAHA, S.; WHITE, G.; WOOLLEN, J.; ZHU, Y.; CHELLIAH, M.; EBISUZAKI, W.; HIGGINS, W.; JANOWIAK, J.; MO, K.C.; ROPELEWSKI, C.; WANG, J.; LEETMAA, A.; REYNOLDS, R.; JOSEPH, D. The NCEP/NCAR 40-year reanalysis project. Bulletin of American Meteorological Society, v. 77, p.437-471, 1996.

KUO, H. -L. Further studies of the parametrization of the influences of cumulus convection on large-scale flow. Journal of the Atmospheric Sciences, v. 31, p. 1232-1240, 1974.

KUO, Y. -H.; REED, R. J. Numerical simulation of an explosively deepening cyclone in the eastern Pacific. Monthly Weather Review, v. 116, p. 2081-2105, 1988.

KUO, Y. -H.; LOW-NAM, S. Prediction of nine explosive cyclones over the western Atlantic ocean with a regional model. Monthly Weather Review, v. 118, p. 3-25, 1990.

KUO, Y. -H., SHAPIRO, M. A.; DONALL, E. G. The interaction between baroclinic and diabiatic processes in a numerical simulation of a rapidly intensifying extratropical marine cyclone. Monthly Weather Review, v. 119, p. 368-384, $1991 \mathrm{a}$.

KUO, Y. -H., REED, R. J.; LOW-NAM, S. Effects of surface energy fluxes during the early development and rapid intensification stages of seven explosive cyclone in the western Atlantic. Monthly Weather Review, v. 119, p. 457 476, $1991 \mathrm{~b}$.

MELLOR, G. L.; YAMADA, T. A hierarchy of turbulence closure models for planetary boundary layers. Journal of the Atmospheric Sciences, v. 31, p. 1791-1806, 1974.

MENDES, D.; SOUZA, E. P.; TRIGO, I. F.; MIRANDA, P. M. A. On precursors of South-American Cyclogenesis. Tellus, v. 59A, p. 114-121, 2007

MILLER, J. E. Cyclogenesis in the Atlantic coastal region of the United States. Journal of Meteorology, v. 3, p. 31-44, 1946.

NAGATA, M.; OGURA, Y.Amodeling case of interaction between heavy precipitation and a low-level jet over Japan in Baiu season. Montlhly Weather Review, v. 119, p. 1309-1336, 1991.

NECCO, G. V. Comportamiento de vortices ciclonicos en el area sudamericana durante el FGGE: Trayectorias y desarrollos. Meteorologica, v. 8, p. 21-34, 1982.

NIELSEN, J. W.; DOLE, R. M. A survey of extratropical cyclone characteristics during GALE. Monthly Weather Review, v. 120, p. 1156-1167, 1992.
NUSS, W. A.; ANTHES, R. A. A numerical investigation of low-level processes in rapid cyclogenesis. Monthly Weather Review, v. 115, p. 2728-2743, 1987.

ODA, T. O. Efeitos da Distribuição Espaço-Temporal da Temperatura do Mar no Desenvolvimento de Ciclones Extratropicais. $125 \mathrm{f}$. Tese (Doutorado em Meteorologia - Universidade Federal do Rio de Janeiro, Rio de Janeiro, 2005.

OGURA, Y. A.; JUANG, H. -M. H. A case study of rapid cyclogenesis over Canada. Part I: Diagnostic study. Monthly Weather Review, v. 118, p. 655-672, 1990.

PIVA, E. D. Estudo de caso sobre o papel dos fluxos de calor latente e sensível em superfície em processos de ciclogênese de costa leste ocorrido na costa da América do Sul. $162 \mathrm{f}$. Dissertação (Mestrado em Meteorologia) - Instituto Nacional de Pesquisas Espaciais, São José dos Campos, 2001.

REBOITA, M. S. ; ROCHA, R. P. da; AMBRIZZI, T. Climatologia de Ciclones sobre o Atlântico Sul utilizando Métodos Objetivos na Detecção destes Sistemas. In: IX CONGRESSO ARGENTINO DE METEOROLOGIA (CONGREMET), 2005. Anais... Buenos Aires, 2005.

REBOITA, M. S. ; ROCHA, R. P. da; AMBRIZZI, T. Effects of Topography and Surface Heat Fluxes on the South Atlantic Extratropical Cyclones: Regional Climate Simulations. In: XXIV GENERAL ASSEMBLY OF THE INTERNATIONAL UNION OF GEODESY AND GEOPHYSICS, 2007, Perugia. Anais... Perugia, 2007.

REED, R. J.; SIMMONS, A. J. Numerical simulation of an explosively deepening cyclone over the north Atlantic that was unaffected by concurrent surface energy fluxes. Weather and Forcasting, v. 6, p. 117-122, 1991.

REYNOLDS, R.W. ; Smith, T.M. Improved Global Sea Surface Temperature Analyses Using Optimum Interpolation. Journal of Climate, v. 7, p. 929-948, 1994.

ROEBBER, P.J. On the statistical analysis of cyclone deepening rates. Monthly Weather Review, v. 117, p. 2293-2298, 1989.

ROCHA, R. P. Impacto de parametrizações de convecção em ciclogênese sobre o oceano. $201 \mathrm{f}$. Tese (Doutorado em Meteorologia) - Instituto Nacional de Pesquisas Espaciais, São José dos Campos, 2000.

SANDERS F.; GYAKUM, J. R. Synoptic-dynamic climatology of the "bomb". Monthly Weather Review, v. 108, p. 15891606, 1980.

SARAIVA, J. M. B. Previsão de Tempo na Região Sul: Efeitos da Liberação de Calor Latente. 205 f. Tese (Doutorado em Meteorologia) - Universidade de São Paulo, São Paulo, 1996. 
SINCLAIR, M.R. An objective cyclone climatology for the Southern Hemisphere. Monthly Weather Review, v. 122, p. 2239-2256, 1994.

SINCLAIR, M.R. A climatology of cyclogenesis for the Southern Hemisphere. Monthly Weather Review, v. 123, p. 1601-1619, 1995.

TATSUMI, Y. An economical explicit time integration scheme for primitive model. Journal Meteorological Society of Japan, v. 61, p. 269-288, 1983.

UCCELLINI, L. W.; PETERSEN, R. A., BRILL, K. F., KOCIN, P. J. E. TUCCILLO, J. J. Synergistic interactions between an upper-level jet streak and diabatic processes that influence the development of a low-level jet and a secondary coastal cyclone. Monthly Weather Review, v. 115, p. 2227-2261, 1987.

UCCELLINI, L. W. Processes contributing to the rapid development of extratropical cyclones. 1992, San Jose. Anais... San Jose, 1992. 8p. (SEM-192L/Doc.2).
YAMAGISHI, Y. Simulation of the air mass transformation processes using a numerical model with the detailed boundary layer parametrization. Journal Meteorological Society of Japan, v. 58, p. 357-377, 1980.

YINLONG, X.; MINGYU, Z. Numerical simulations on the explosive cyclogenesis over the Kuroshio current. Advanced Atmospheric Science, v. 16, p. 64-76, 1999.

YOSHIDA, A.; ASUMA, Y. Structures and Environment of Explosively Developing Extratropical Cyclones in the Northwestern Pacific Region. Monthly Weather Review, v. 132, p. 1121-1142, 2004.

WASH, C. H. HALO, R. A., DOBOS, P. H.; WRIGHT, E. J. Study of explosive and nonexplosive cyclogenesis during FGGE. Monthly Weather Review, v. 120, p. 40-51, 1992. 